\title{
Compressed Conjugacy and the Word Problem for Outer Automorphism Groups of Graph Groups
}

\author{
Niko Haubold, Markus Lohrey, and Christian Mathissen \\ Institut für Informatik, Universität Leipzig, Germany \\ \{haubold, lohrey, mathissen\}@informatik.uni-leipzig.de
}

\begin{abstract}
It is shown that for graph groups (right-angled Artin groups) the conjugacy problem as well as a restricted version of the simultaneous conjugacy problem can be solved in polynomial time even if input words are represented in a compressed form. As a consequence it follows that the word problem for the outer automorphism group of a graph group can be solved in polynomial time.
\end{abstract}

\section{Introduction}

Automorphism groups and outer automorphism groups of graph groups received a lot of interest in the past few years. A graph group $\mathbb{G}(\Sigma, I)$ is given by a finite undirected graph $(\Sigma, I)$ (without self-loops). The set $\Sigma$ is the set of generators of $\mathbb{G}(\Sigma, I)$ and every edge $(a, b) \in I$ gives rise to a commutation relation $a b=b a$. Graph groups are also known as right-angled Artin groups or free partially commutative groups. Graph groups interpolate between finitely generated free groups and finitely generated free Abelian groups. The automorphism group of the free Abelian group $\mathbb{Z}^{n}$ is $\mathrm{GL}(n, \mathbb{Z})$ and hence finitely generated. By a classical result of Nielsen, also automorphism groups of free groups are finitely generated, see e.g. [19]. For graph groups in general, it was shown by Laurence [14] (building up on previous work by Servatius [27]) that their automorphism groups are finitely generated. Only recently, Day [8] has shown that $\operatorname{Aut}(\mathbb{G}(\Sigma, I))$ is always finitely presented. Some recent structural results on automorphism groups of graph groups can be found in [4,5]; for a survey see [3].

In this paper, we continue the investigation of algorithmic aspects of automorphism groups of graph groups. In [18] it was shown that the word problem for Aut $(\mathbb{G}(\Sigma, I))$ can be solved in polynomial time. The proof of this result used compression techniques. It is well-known that the word problem for $\mathbb{G}(\Sigma, I)$ can be solved in linear time. In [18], a compressed (or succinct) version of the word problem for graph groups was studied. In this variant of the word problem, the input word is represented succinctly by a so-called straight-line program. This is a context free grammar $\mathbb{A}$ that generates exactly one word $\operatorname{val}(\mathbb{A})$, see Section 2.1. Since the length of this word may grow exponentially with the size (number of productions) of the SLP $\mathbb{A}$, SLPs can be seen indeed as a succinct string representation. SLPs turned out to be a very flexible compressed representation of strings, which are well suited for studying algorithms for compressed data, see e.g. [11, $15,17,21,23,24]$. In $[18,26]$ it was shown that the word problem for the automorphism group Aut $(G)$ of a group $G$ can be reduced in polynomial time to the compressed word problem for $G$, where the input word is succinctly given by an SLP. In [26], it was 
shown that the compressed word problem for a finitely generated free group $F$ can be solved in polynomial time and in [18] this result was extended to graph groups. It follows that the word problem for Aut $(\mathbb{G}(\Sigma, I))$ can be solved in polynomial time. Recently, Macdonald [20] has shown that also the compressed word problem for every fully residually free group can be solved in polynomial time.

It is not straightforward to carry over the above mentioned complexity results from Aut $(\mathbb{G}(\Sigma, I))$ to the outer automorphism group

$$
\operatorname{Out}(\mathbb{G}(\Sigma, I))=\operatorname{Aut}(\mathbb{G}(\Sigma, I)) / \operatorname{Inn}(\mathbb{G}(\Sigma, I)) .
$$

Nevertheless, Schleimer proved in [26] that the word problem for the outer automorphism group of a finitely generated free group can be decided in polynomial time. For this, he used a compressed variant of the simultaneous conjugacy problem in free groups. In this paper, we generalize Schleimer's result to graph groups: For every graph $(\Sigma, I)$, the word problem for Out $(\mathbb{G}(\Sigma, I))$ can be solved in polynomial time. Analogously to Schleimer's approach for free groups, we reduce the word problem for Out $(\mathbb{G}(\Sigma, I))$ to a compressed variant of the simultaneous conjugacy problem in $\mathbb{G}(\Sigma, I)$. In this problem, we have given an SLP $\mathbb{A}_{a}$ for every generator $a \in \Sigma$, and the question is whether there exists $x \in \mathbb{G}(\Sigma, I)$ such that $a=x \operatorname{val}\left(\mathbb{A}_{a}\right) x^{-1}$ for all $a \in \Sigma$. A large part of this paper develops a polynomial time algorithm for this problem. Moreover, we also present a polynomial time algorithm for the compressed version of the classical conjugacy problem in graph groups: In this problem, we have given two SLPs $\mathbb{A}$ and $\mathbb{B}$ and we ask whether there exists $x \in \mathbb{G}(\Sigma, I)$ such that $\operatorname{val}(\mathbb{A})=x \operatorname{val}(\mathbb{B}) x^{-1}$ in $\mathbb{G}(\Sigma, I)$. For our polynomial time algorithm, we have to develop a pattern matching algorithm for SLP-compressed Mazurkiewicz traces, which is inspired by a pattern matching algorithm for hierarchical message sequence charts from [12]. For the noncompressed version of the conjugacy problem in $\mathbb{G}(\Sigma, I)$, a linear time algorithm was presented in [29] based on [16]. In [7] this result was generalized to various subgroups of graph groups.

\section{Preliminaries}

Let $\Sigma$ be a finite alphabet. For a word $s=a_{1} \cdots a_{m}\left(a_{i} \in \Sigma\right)$ let

$$
\begin{aligned}
& \text { - }|s|=m, \text { alph }(s)=\left\{a_{1}, \ldots, a_{m}\right\}, \\
& \text { - } s[i]=a_{i} \text { for } 1 \leq i \leq m, \\
& \text { - } s[i: j]=a_{i} \cdots a_{j} \text { for } 1 \leq i \leq j \leq m \text { and } s[i: j]=\varepsilon \text { for } i>j, \text { and } \\
& \text { - }|s|_{a}=|\{k \mid s[k]=a\}| \text { for } a \in \Sigma .
\end{aligned}
$$

We use $\Sigma^{-1}=\left\{a^{-1} \mid a \in \Sigma\right\}$ to denote a disjoint copy of $\Sigma$ and let $\Sigma^{ \pm 1}=\Sigma \cup \Sigma^{-1}$. Define $\left(a^{-1}\right)^{-1}=a$; this defines an involution ${ }^{-1}: \Sigma^{ \pm 1} \rightarrow \Sigma^{ \pm 1}$, which can be extended to an involution on $\left(\Sigma^{ \pm 1}\right)^{*}$ by setting $\left(a_{1} \cdots a_{n}\right)^{-1}=a_{n}^{-1} \cdots a_{1}^{-1}$.

\subsection{Straight-line programs}

We are using straight-line programs as a succinct representation of strings with reoccurring subpatterns [25]. A straight-line program (SLP) over the alphabet $\Gamma$ is a context 
free grammar $\mathbb{A}=(V, \Gamma, S, P)$, where $V$ is the set of nonterminals, $\Gamma$ is the set of terminals, $S \in V$ is the initial nonterminal, and $P \subseteq V \times(V \cup \Gamma)^{*}$ is the set of productions such that (i) for every $X \in V$ there is exactly one $\alpha \in(V \cup \Gamma)^{*}$ with $(X, \alpha) \in P$ and (ii) there is no cycle in the relation $\{(X, Y) \in V \times V \mid \exists \alpha:(X, \alpha) \in P, Y \in \operatorname{alph}(\alpha)\}$. These conditions ensure that the language generated by the straight-line program $\mathbb{A}$ contains exactly one word $\operatorname{val}(\mathbb{A})$. Moreover, every nonterminal $X \in V$ generates exactly one word that is denoted by $\operatorname{val}_{\mathbb{A}}(X)$, or briefly $\operatorname{val}(X)$, if $\mathbb{A}$ is clear from the context. The size of $\mathbb{A}$ is $|\mathbb{A}|=\sum_{(X, \alpha) \in P}|\alpha|$. It can be seen easily that an SLP can be transformed in polynomial time into an equivalent SLP in Chomsky normal form, which means that all productions have the form $A \rightarrow B C$ or $A \rightarrow a$ with $A, B, C \in V$ and $a \in \Gamma$.

For an SLP $\mathbb{A}$ over $\Sigma^{ \pm 1}$ (w.l.o.g. in Chomsky normal form) we denote with $\mathbb{A}^{-1}$ the SLP that has for each terminal rule $A \rightarrow a$ from $\mathbb{A}$ the terminal rule $A \rightarrow a^{-1}$ and for each nonterminal rule $A \rightarrow B C$ from $\mathbb{A}$ the nonterminal rule $A \rightarrow C B$. Clearly, $\operatorname{val}\left(\mathbb{A}^{-1}\right)=\operatorname{val}(\mathbb{A})^{-1}$.

Let us state some simple algorithmic problems that can be easily solved in polynomial time:

- Given an SLP A, calculate $|\operatorname{val}(\mathbb{A})|$.

- Given an SLP $\mathbb{A}$ and a number $i \in\{1, \ldots,|\operatorname{val}(\mathbb{A})|\}$, calculate val $(\mathbb{A})[i]$.

- Given an SLP $\mathbb{A}$ and two numbers $1 \leq i \leq j \leq|\operatorname{val}(\mathbb{A})|$, compute and SLP $\mathbb{B}$ with $\operatorname{val}(\mathbb{B})=\operatorname{val}(\mathbb{A})[i, j]$.

In [23], Plandowski presented a polynomial time algorithm for testing whether val $(\mathbb{A})=$ $\operatorname{val}(\mathbb{B})$ for two given SLPs $\mathbb{A}$ and $\mathbb{B}$. A cubic algorithm was presented by Lifshits [15]. In fact, Lifshits gave an algorithm for compressed pattern matching: given SLPs $\mathbb{A}$ and $\mathbb{B}$, is $\mathbb{A}$ a factor of $\mathbb{B}$ ? The running time of his algorithm is $O\left(|\mathbb{A}| \cdot|\mathbb{B}|^{2}\right)$.

A composition system $\mathbb{A}=(V, \Gamma, S, P)$ is defined analogously to an SLP, but in addition to productions of the form $A \rightarrow \alpha\left(A \in V, \alpha \in(V \cup \Gamma)^{*}\right)$ it may also contain productions of the form $A \rightarrow B[i: j]$ for $B \in V$ and $i, j \in \mathbb{N}[11]$. For such a production we define $\operatorname{val}_{\mathbb{A}}(A)=\operatorname{val}_{\mathbb{A}}(B)[i: j]$. The size of a production $A \rightarrow B[i: j]$ is $\lceil\log (i)\rceil+\lceil\log (j)\rceil$. As for SLPs we define val $(\mathbb{A})=\operatorname{val}_{\mathbb{A}}(S)$. In [13], Hagenah presented a polynomial time algorithm, which transforms a given composition system $\mathbb{A}$ into an SLP $\mathbb{B}$ with $\operatorname{val}(\mathbb{A})=\operatorname{val}(\mathbb{B})$. Composition systems were also heavily used in $[18,26]$ in order to solve compressed word problems efficiently.

\subsection{Trace monoids and graph groups}

We introduce some notions from trace theory. For a thorough introduction see [10]. An independence alphabet is a finite undirected graph $(\Sigma, I)$ without loops. Then $I \subseteq$ $\Sigma \times \Sigma$ is an irreflexive and symmetric relation. The complementary graph $(\Sigma, D)$ with $D=(\Sigma \times \Sigma) \backslash I$ is called a dependence alphabet. The trace monoid $\mathbb{M}(\Sigma, I)$ is defined as the quotient $\mathbb{M}(\Sigma, I)=\Sigma^{*} /\{a b=b a \mid(a, b) \in I\}$ with concatenation as operation and the empty word as the neutral element. This monoid is cancellative and its elements are called traces. The trace represented by the word $s \in \Sigma^{*}$ is denoted by $[s]_{I}$. As an ease of notation we denote with $u \in \Sigma^{*}$ also the trace $[u]_{I}$. For $a \in \Sigma$ let 
$I(a)=\{b \in \Sigma \mid(a, b) \in I\}$ be the letters that commute with $a$ and $D(a)=\{b \in$ $\Sigma \mid(a, b) \in D\}$ be the letters that are dependent from $a$. For traces $u, v \in \mathbb{M}(\Sigma, I)$ we denote with $u I v$ the fact that $\operatorname{alph}(u) \times \operatorname{alph}(v) \subseteq I$. For $\Gamma \subseteq \Sigma$ we say that $\Gamma$ is connected if the subgraph of $(\Sigma, D)$ induced by $\Gamma$ is connected. For a trace $u$ we denote with $\max (u)$ the set of possible last letters of $u$, i.e., $\max (u)=\{a \mid u=v a$ for $a \in$ $\Sigma, v \in \mathbb{M}(\Sigma, I)\}$. Analogously we define $\min (u)$ to be the set of possible first letters i.e., $\min (u)=\{a \mid u=a v$ for $a \in \Sigma, v \in \mathbb{M}(\Sigma, I)\}$.

A convenient representation for traces are dependence graphs, which are nodelabeled directed acyclic graphs. For a word $w \in \Sigma^{*}$ the dependence graph $D_{w}$ has vertex set $\{1, \ldots,|w|\}$ where the node $i$ is labeled with $w[i]$. There is an edge from vertex $i$ to $j$ if and only if $i<j$ and $(w[i], w[j]) \in D$. It is easy to see that for two words $w, w^{\prime} \in \Sigma^{*}$ we have $[w]_{I}=\left[w^{\prime}\right]_{I}$ if and only if $D_{w}$ and $D_{w^{\prime}}$ are isomorphic. Hence, we can speak of the dependence graph of a trace.

For background in combinatorial group theory see [19]. The free group $F(\Sigma)$ generated by $\Sigma$ can be defined as the quotient monoid

$$
F(\Sigma)=\left(\Sigma^{ \pm 1}\right)^{*} /\left\{a a^{-1}=\varepsilon \mid a \in \Sigma^{ \pm 1}\right\} .
$$

For an independence alphabet $(\Sigma, I)$ the graph group $\mathbb{G}(\Sigma, I)$ is defined as the quotient group

$$
\mathbb{G}(\Sigma, I)=F(\Sigma) /\{a b=b a \mid(a, b) \in I\} .
$$

From the independence alphabet $(\Sigma, I)$ we derive the independence alphabet

$$
\left(\Sigma^{ \pm 1},\left\{\left(a^{\varepsilon_{1}}, b^{\varepsilon_{2}}\right) \mid(a, b) \in I, \varepsilon_{1}, \varepsilon_{2} \in\{-1,1\}\right\}\right) .
$$

Abusing notation, we denote the independence relation of this alphabet again with $I$.

Note that $(a, b) \in I$ implies $a^{-1} b=b a^{-1}$ in $\mathbb{G}(\Sigma, I)$. Thus, the graph group $\mathbb{G}(\Sigma, I)$ can be also defined as the quotient

$$
\mathbb{G}(\Sigma, I)=\mathbb{M}\left(\Sigma^{ \pm 1}, I\right) /\left\{a a^{-1}=\varepsilon \mid a \in \Sigma^{ \pm 1}\right\} .
$$

Graph groups are also known as right-angled Artin groups and free partially commutative groups.

\section{3 (Outer) automorphism groups}

The automorphism group $\operatorname{Aut}(G)$ of a group $G$ is the set of all bijective homomorphisms from $G$ to itself with composition as operation and the identity mapping as the neutral element. An automorphism $\varphi$ is called inner if there is a group element $x \in G$ such that $\varphi(y)=x y x^{-1}$ for all $y \in G$. The set of all inner automorphisms for a group $G$ forms the inner automorphism group of $G$ denoted by $\operatorname{lnn}(G)$. This is easily seen to be a normal subgroup of $\operatorname{Aut}(G)$ and the quotient group $\operatorname{Out}(G)=\operatorname{Aut}(G) / \operatorname{lnn}(G)$ is called the outer automorphism group of $G$.

Assume that $\operatorname{Aut}(G)$ is finitely generated ${ }^{1}$ and let $\Psi=\left\{\psi_{1}, \ldots, \psi_{k}\right\}$ be a monoid generating set for Aut $(G)$. Then $\Psi$ also generates $\operatorname{Out}(G)$ where we identify $\psi_{i}$ with the

\footnotetext{
${ }^{1}$ In general, this won't be the case, even if $G$ is finitely generated.
} 
$\operatorname{coset} \psi_{i} \operatorname{Inn}(G)$ for $i \in\{1, \ldots, k\}$. Then the word problem for the outer automorphism group can be viewed as the following decision problem:

INPUT: A word $w \in \Psi^{*}$.

QUESTION: Does $w=1$ in $\operatorname{Out}(G)$ ?

Since an automorphism belongs to the same coset (with respect to $\operatorname{lnn}(G)$ ) as the identity if and only if it is inner, we can rephrase the word problem for Out $(G)$ as follows:

INPUT: A word $w \in \Psi^{*}$.

QUESTION: Does $w$ represent an element of $\operatorname{Inn}(G)$ in $\operatorname{Aut}(G)$ ?

Building on results from [27], Laurence has shown in [14] that automorphism groups of graph groups are finitely generated. Recently, Day [8] proved that automorphism groups of graph groups are in fact finitely presented. Further results on (outer) automorphism groups of graph groups can be found in $[4,5]$. The main purpose of this paper is to give a polynomial time algorithm for the word problem for $\operatorname{Out}(\mathbb{G}(\Sigma, I))$.

\section{Main results}

In this section we will present the main results of this paper the proof of which are subject to the rest of the paper. In order to solve the word problem for Out $(\mathbb{G}(\Sigma, I))$ in polynomial time, we have to deal with compressed conjugacy problems in $\mathbb{G}(\Sigma, I)$. Recall that two elements $g$ and $h$ of a group $G$ are conjugated if and only if there exists $x \in G$ such that $g=x h x^{-1}$. The classical conjugacy problem for $G$ asks, whether two given elements of $G$ are conjugated. We will consider a compressed variant of this problem in $\mathbb{G}(\Sigma, I)$, which we call the compressed conjugacy problem for $\mathbb{G}(\Sigma, I)$, $\operatorname{CCP}(\Sigma, I)$ for short:

INPUT: SLPs $\mathbb{A}$ and $\mathbb{B}$ over $\Sigma^{ \pm 1}$. QUESTION: $\operatorname{Are} \operatorname{val}(\mathbb{A})$ and $\operatorname{val}(\mathbb{B})$ conjugated in $\mathbb{G}(\Sigma, I)$ ?

Theorem 1. Let $(\Sigma, I)$ be a fixed independence alphabet. Then, $\operatorname{CCP}(\Sigma, I)$ can be solved in polynomial time.

We will proof Theorem 1 in Section 10.

In order to solve the word problem for $\operatorname{Out}(\mathbb{G}(\Sigma, I))$ in polynomial time, Theorem 1 is not sufficient. We need an extension of $\operatorname{CCP}(\Sigma, I)$ to several pairs of input SLPs. Let us call this problem the simultaneous compressed conjugacy problem for $\mathbb{G}(\Sigma, I)$ :

INPUT: SLPs $\mathbb{A}_{1}, \mathbb{B}_{1}, \ldots, \mathbb{A}_{n}, \mathbb{B}_{n}$ over $\Sigma^{ \pm 1}$.

QUESTION: Does there exist $x \in\left(\Sigma^{ \pm 1}\right)^{*}$ such that $\operatorname{val}\left(\mathbb{A}_{i}\right)=x \operatorname{val}\left(\mathbb{B}_{i}\right) x^{-1}$ in $\mathbb{G}(\Sigma, I)$ for all $i \in\{1, \ldots, n\}$ ?

The simultaneous (non-compressed) conjugacy problem also appears in connection with group-based cryptography [22]. Unfortunately, we don't know, whether the simultaneous compressed conjugacy problem can be solved in polynomial time. But, in order to deal with the word problem for Out $(\mathbb{G}(\Sigma, I))$, a restriction of this problem suffices, 
where the SLPs $\mathbb{B}_{1}, \ldots, \mathbb{B}_{n}$ from the simultaneous compressed conjugacy problem are the letters from $\Sigma$. We call this problem the restricted simultaneous compressed conjugacy problem, briefly $\operatorname{RSCCP}(\Sigma, I)$ :

INPUT: SLPs $\mathbb{A}_{a}(a \in \Sigma)$ over $\Sigma^{ \pm 1}$.

QUESTION: Does there exist $x \in\left(\Sigma^{ \pm 1}\right)^{*}$ with $\operatorname{val}\left(\mathbb{A}_{a}\right)=x a x^{-1}$ in $\mathbb{G}(\Sigma, I)$ for all $a \in \Sigma$ ?

An $x$ such that $\operatorname{val}\left(\mathbb{A}_{a}\right)=x a x^{-1}$ in $\mathbb{G}(\Sigma, I)$ for all $a \in \Sigma$ is called a solution of the $\operatorname{RSCCP}(\Sigma, I)$-instance. The following theorem will be shown in Section 7.

Theorem 2. Let $(\Sigma, I)$ be a fixed independence alphabet. Then, $\operatorname{RSCCP}(\Sigma, I)$ can be solved in polynomial time. Moreover, in case a solution exists, one can compute an SLP for a solution in polynomial time.

Using Theorem 2, it is straightforward to decide the word problem for Out $(\mathbb{G}(\Sigma, I))$ in polynomial time.

Theorem 3. Let $(\Sigma, I)$ be a fixed independence alphabet. Then, the word problem for the group Out $(\mathbb{G}(\Sigma, I))$ can be solved in polynomial time.

Proof. Fix a finite monoid generating set $\Phi$ for $\operatorname{Aut}(\mathbb{G}(\Sigma, I))$. Let $\varphi=\varphi_{1} \cdots \varphi_{n}$ with $\varphi_{1}, \ldots, \varphi_{n} \in \Phi$ be the input. By [26] we can compute in polynomial time SLPs $\mathbb{A}_{a}$ $(a \in \Sigma)$ over $\Sigma^{ \pm 1}$ with $\operatorname{val}\left(\mathbb{A}_{a}\right)=\varphi(a)$ in $\mathbb{G}(\Sigma, I)$ for all $a \in \Sigma$. The automorphism $\varphi$ is inner if and only if there exists $x$ such that $\operatorname{val}\left(\mathbb{A}_{a}\right)=x^{-1}$ in $\mathbb{G}(\Sigma, I)$ for all $a \in \Sigma$. This can be decided in polynomial time by Theorem 2 .

It is important in Theorem 1-3 that we fix the independence alphabet $(\Sigma, I)$. It is open whether these results also hold if $(\Sigma, I)$ is part of the input.

\section{Simple facts for traces}

In this section, we state some simple facts on the prefix order of trace monoids, which will be needed later. A trace $u$ is said to be a prefix of a trace $w$ if there exists a trace $v$ such that $u v=w$ and we denote this fact by $u \preceq w$. The prefixes of a trace $w$ correspond to the downward-closed node sets of the dependence graph of $w$. Analogously a trace $v$ is a suffix of a trace $w$ if there is a trace $u$ such that $u v=w$. For two traces $u, v \in \mathbb{M}(\Sigma, I)$, the infimum $u \sqcap v$ is the largest trace $s$ with respect to $\preceq$ such that $s \preceq u$ and $s \preceq v$; it always exists [6]. With $u \backslash v$ we denote the unique trace $t$ such that $u=(u \sqcap v) t$; uniqueness follows from the fact that $\mathbb{M}(\Sigma, I)$ is cancellative. Note that $u \backslash v=u \backslash(u \sqcap v)$.

The supremum $u \sqcup v$ of two traces $u, v \in \mathbb{M}(\Sigma, I)$ is the smallest trace $s$ with respect to $\preceq$ such that $u \preceq s$ and $v \preceq s$ if any such trace exists. The following result can be found in [6]:

Lemma 4 ([6]). The trace $u \sqcup v$ exists if and only if $(u \backslash v) I(v \backslash u)$, in which case we have $u \sqcup v=(u \sqcap v)(u \backslash v)(v \backslash u)$. 
We can define the supremum of several traces $w_{1}, \ldots, w_{n}$ analogously by induction: $w_{1} \sqcup \cdots \sqcup w_{n}=\left(w_{1} \sqcup \cdots \sqcup w_{n-1}\right) \sqcup w_{n}$. We mention a necessary and sufficient condition for the existence of the supremum of several traces that follows directly from the definition.

Lemma 5. Let $(\Sigma, I)$ be an independence alphabet and $u_{1}, \ldots, u_{r} \in \mathbb{M}(\Sigma, I)$. If $u=$ $u_{1} \sqcup \cdots \sqcup u_{r-1}$ exists then $s=u_{1} \sqcup \cdots \sqcup u_{r}$ is exists if and only if $\left(u \backslash u_{r}\right) I\left(u_{r} \backslash u\right)$. In this case $s=u\left(u_{r} \backslash u\right)$.

Example 6. We consider the following independence alphabet $(\Sigma, I)$ :

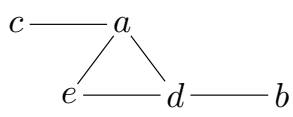

Then the corresponding dependence alphabet is:

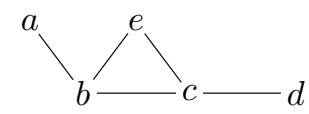

We consider the words $u=$ aeadbacdd and $v=e a a b d c a e b$. Then the dependence graphs $D_{u}$ of $u$ and $D_{v}$ of $v$ look as follows (where we label the vertices $i$ with the letter $u[i]$ (resp. $v[i])$ ):
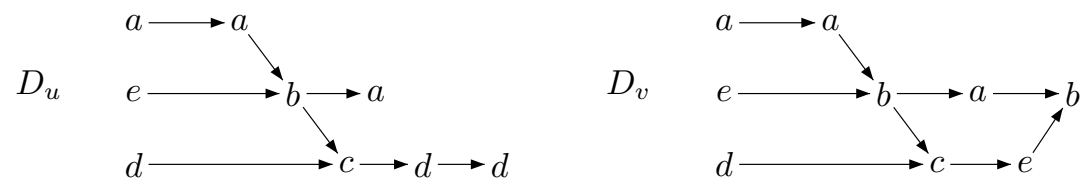

Then we have $u \sqcap v=a e a d b a c=: p$ and its dependence graph is:

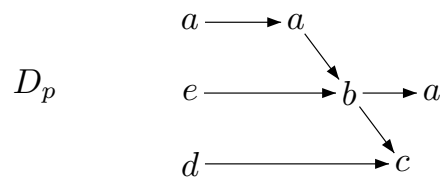

Since $u \backslash p=d d$ and $v \backslash p=e b$ we have $(u \backslash p) I(v \backslash p)$ and hence the supremum $s=u \sqcup v=a e a d b a c d d e b$ is defined. The dependence graph for $s$ is:

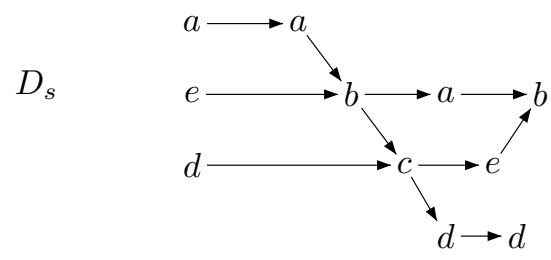

The following lemma is a basic statement for traces, see for example [10]: 
Lemma 7 (Levi's Lemma). Let $u_{1}, u_{2}, v_{1}, v_{2}$ be traces such that $u_{1} u_{2}=v_{1} v_{2}$. Then there exist traces $x, y_{1}, y_{2}, z$ such that $y_{1} I y_{2}$ and $u_{1}=x y_{1}, u_{2}=y_{2} z, v_{1}=x y_{2}$, and $v_{2}=y_{1} z$.

We use Levi's Lemma to prove the following statement:

Lemma 8. Let $a \in \Sigma$. The decomposition of a trace $t \in \mathbb{M}(\Sigma, I)$ as $t=u_{1} u_{2}$ with $u_{2} I a$ and $\left|u_{2}\right|$ maximal is unique in $\mathbb{M}(\Sigma, I)$.

Proof. Let $u_{1} u_{2}=t=v_{1} v_{2}$ be such that $u_{2} I a, v_{2} I a$ and $\left|u_{2}\right|$ and $\left|v_{2}\right|$ are both maximal (hence $\left.\left|u_{2}\right|=\left|v_{2}\right|\right)$. By Levi's Lemma there are traces $x, y_{1}, y_{2}, z$ such that $y_{1} I y_{2}$ and $u_{1}=x y_{1}, u_{2}=y_{2} z, v_{1}=x y_{2}$, and $v_{2}=y_{1} z$. From $u_{2} I a$ and $v_{2} I a$ we get $y_{1} I a$ and $y_{2} I a$. Maximality of $\left|u_{2}\right|=\left|v_{2}\right|$ and $x y_{1} u_{2}=t=x y_{2} v_{2}$ implies $y_{1}=y_{2}=\varepsilon$. Hence $u_{1}=v_{1}$ and $u_{2}=v_{2}$.

A trace rewriting system $R$ over $\mathbb{M}(\Sigma, I)$ is just a finite subset of $\mathbb{M}(\Sigma, I) \times \mathbb{M}(\Sigma, I)$ [9]. We can define the one-step rewrite relation $\rightarrow_{R} \subseteq \mathbb{M}(\Sigma, I) \times \mathbb{M}(\Sigma, I)$ by: $x \rightarrow_{R} y$ if and only if there are $u, v \in \mathbb{M}(\Sigma, I)$ and $(\ell, r) \in R$ such that $x=u \ell v$ and $y=u r v$. With $\stackrel{*}{\rightarrow}_{R}$ we denote the reflexive transitive closure of $\rightarrow_{R}$. The notion of a confluent and terminating trace rewriting system is defined as for other types of rewriting systems [2]: A trace rewriting system $R$ is called confluent if for all $u, v, v^{\prime} \in \mathbb{M}(\Sigma, I)$ it holds that $u \stackrel{*}{\rightarrow}_{R} v$ and $u \stackrel{*}{\rightarrow}_{R} v^{\prime}$ imply that there is a trace $w$ with $v \stackrel{*}{\rightarrow}_{R} w$ and $v^{\prime} \stackrel{*}{\rightarrow}_{R} w$. It is called terminating if there does not exist an infinite chain $u_{0} \rightarrow_{R} u_{1} \rightarrow_{R} u_{2} \cdots$. A trace $u$ is $R$-irreducible if no trace $v$ with $u \rightarrow_{R} v$ exists. The set of all $R$-irreducible traces is denoted with $\operatorname{IRR}(R)$. If $R$ is terminating and confluent, then for every trace $u$, there exists a unique normal form $\mathrm{NF}_{R}(u) \in \operatorname{IRR}(R)$ such that $u \stackrel{*}{\rightarrow}_{R} \mathrm{NF}_{R}(u)$.

Let us now work in the trace monoid $\mathbb{M}\left(\Sigma^{ \pm 1}, I\right)$. For a trace $u=\left[a_{1} \cdots a_{n}\right]_{I} \in$ $\mathbb{M}\left(\Sigma^{ \pm 1}, I\right)$ we denote with $u^{-1}$ the trace $u^{-1}=\left[a_{n}^{-1} \cdots a_{1}^{-1}\right]_{I}$. It is easy to see that this definition is independent of the chosen representative $a_{1} \cdots a_{n}$ of the trace $u$. It follows that we have $[\operatorname{val}(\mathbb{A})]_{I}^{-1}=\left[\operatorname{val}\left(\mathbb{A}^{-1}\right)\right]_{I}$ for an SLP $\mathbb{A}$. For the rest of the paper, we fix the trace rewriting system

$$
R=\left\{\left(\left[a a^{-1}\right]_{I},[\varepsilon]_{I}\right) \mid a \in \Sigma^{ \pm 1}\right\}
$$

over the trace monoid $\mathbb{M}\left(\Sigma^{ \pm 1}, I\right)$. Since $R$ is length-reducing, $R$ is terminating. By [ 9 , 28], $R$ is also confluent. For traces $u, v \in \mathbb{M}\left(\Sigma^{ \pm 1}, I\right)$ we have $u=v$ in $\mathbb{G}(\Sigma, I)$ if and only if $\mathrm{NF}_{R}(u)=\mathrm{NF}_{R}(v)$. Using these facts, it was shown in $[9,28]$ that the word problem for $\mathbb{G}(\Sigma, I)$ can be solved in linear time (on the RAM model).

\section{Algorithms for compressed traces}

In this section, we will recall some results from [18] concerning traces, which are represented by SLPs. For SLPs $\mathbb{A}$ and $\mathbb{B}$ over $\Sigma^{ \pm 1}$ we say that $\mathbb{B}$ is an $R$-reduction of $\mathbb{A}$ if $[\operatorname{val}(\mathbb{B})]_{I}=\mathrm{NF}_{R}\left([\operatorname{val}(\mathbb{A})]_{I}\right)$. We will need the following theorem.

Theorem 9 ([18]). Let $\mathbb{A}$ be an SLP over $\Sigma^{ \pm 1}$ representing a trace in $\mathbb{M}\left(\Sigma^{ \pm 1}, I\right)$. We can compute an $R$-reduction for $\mathbb{A}$ in polynomial time. 
Corollary 10. The following decision problem can be solved in polynomial time.

INPUT: An SLP $\mathbb{A}$ over $\Sigma^{ \pm 1}$.

QUESTION: $\mathrm{NF}_{R}\left([\operatorname{val}(\mathbb{A})]_{I}\right)=[\varepsilon]_{I}$ ?

Note that this is equivalent to a polynomial time solution of the compressed word problem for graph groups.

Theorem 11 ([18]). For given SLPs $\mathbb{A}_{0}$ and $\mathbb{A}_{1}$ over $\Sigma^{ \pm 1}$, we can compute in polynomial time SLPs $\mathbb{P}, \mathbb{D}_{0}, \mathbb{D}_{1}$ with $[\operatorname{val}(\mathbb{P})]_{I}=\left[\operatorname{val}\left(\mathbb{A}_{0}\right)\right]_{I} \sqcap\left[\operatorname{val}\left(\mathbb{A}_{1}\right)\right]_{I}$ and $\left[\operatorname{val}\left(\mathbb{D}_{i}\right)\right]_{I}=$ $\left[\operatorname{val}\left(\mathbb{A}_{i}\right)\right]_{I} \backslash\left[\operatorname{val}\left(\mathbb{A}_{1-i}\right)\right]_{I}(i \in\{0,1\})$.

An immediate corollary of Theorem 11 and Lemma 4 is:

Corollary 12. For given SLPS $\mathbb{A}_{0}$ and $\mathbb{A}_{1}$ over $\Sigma^{ \pm 1}$, we can check in polynomial time, whether $\left[\operatorname{val}\left(\mathbb{A}_{0}\right)\right]_{I} \sqcup\left[\operatorname{val}\left(\mathbb{A}_{1}\right)\right]_{I}$ exists, and in case it exists, we can compute in polynomial time an $S L P \mathbb{S}$ with $[\operatorname{val}(\mathbb{S})]_{I}=\left[\operatorname{val}\left(\mathbb{A}_{0}\right)\right]_{I} \sqcup\left[\operatorname{val}\left(\mathbb{A}_{1}\right)\right]_{I}$.

Lemma 5 and Corollary 12 imply the following corollary.

Corollary 13. Let $r$ be a fixed constant. For given $S L P s \mathbb{V}_{1}, \ldots, \mathbb{V}_{r}$ over $\Sigma^{ \pm 1}$, we can decide in polynomial time whether $\left[\mathrm{val}\left(\mathbb{V}_{1}\right)\right]_{I} \sqcup \cdots \sqcup\left[\mathrm{val}\left(\mathbb{V}_{r}\right)\right]_{I}$ exists, and in case it exists we can compute in polynomial time an $S L P \mathbb{S}$ with $[\operatorname{val}(\mathbb{S})]_{I}=\left[\operatorname{val}\left(\mathbb{V}_{1}\right)\right]_{I} \sqcup \cdots \sqcup$ $\left[\operatorname{val}\left(\mathbb{V}_{r}\right)\right]_{I}$

It is important that we fix the number $r$ of SLPs in Corollary 13: Each application of Lemma 12 may increase the size of the SLP polynomially. Hence, a non-fixed number of applications might lead to an exponential blow-up.

\section{Double $a$-cones}

The definition of the problem $\operatorname{RSCCP}(\Sigma, I)$ in Section 3 motivates the following definition: A double a-cone for $a \in \Sigma^{ \pm 1}$ is an $R$-irreducible trace of the form $u a u^{-1}$ with $u \in \mathbb{M}\left(\Sigma^{ \pm 1}, I\right)$. In this section, we will prove several results on double $a$-cones, which will be needed later for deciding $\operatorname{RSCCP}(\Sigma, I)$ in polynomial time.

Lemma 14. A trace $u a u^{-1}$ is a double a-cone if and only if the following conditions hold:

(1) $u \in \operatorname{IRR}(R)$

(2) $\max (u) \cap\left(\left\{a, a^{-1}\right\} \cup I(a)\right)=\emptyset$.

Proof. Let $v=u a u^{-1}$ be a double $a$-cone. Since $v \in \operatorname{IRR}(R)$, also $u \in \operatorname{IRR}(R)$. If $a^{\varepsilon} \in \max (u)$ for $\varepsilon \in\{1,-1\}$ then $v=u^{\prime} a^{\varepsilon} a a^{-\varepsilon} u^{\prime-1}$ for some trace $u^{\prime}$ contradicting the $R$-irreducibility of $v$. Similarly, if there is some $b \in I(a) \cap \max (u)$ it follows that $v=u^{\prime} b a b^{-1} u^{\prime-1}=u^{\prime} a b b^{-1} u^{\prime-1}$ again a contradiction. Suppose on the other hand that (1) and (2) hold for $v=u a u^{-1}$. Since $u \in \operatorname{IRR}(R)$ and no element from $\max (u)$ cancels against or commutes with $a$ it follows that $v$ is also $R$-irreducible. 
It follows that every letter in a double $a$-cone either lies before or after the central letter $a$. Its dependence graph always has the following form:

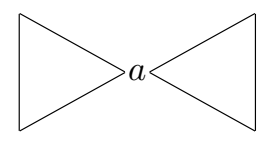

By the following lemma, each double $a$-cone has a unique factorization of the form $u_{1} b u_{2}$ with $\left|u_{1}\right|=\left|u_{2}\right|$.

Lemma 15. Let $v=u a u^{-1}$ be a double $a$-cone and let $v=u_{1} b u_{2}$ with $b \in \Sigma^{ \pm 1}$ and $\left|u_{1}\right|=\left|u_{2}\right|$. Then $a=b, u_{1}=u$ and $u_{2}=u^{-1}$.

Proof. Let $v=u a u^{-1}=u_{1} b u_{2}$ be a double $a$-cone where $\left|u_{1}\right|=\left|u_{2}\right|$. We have $\max (u a)=\{a\}$ and $(a, c) \in D$ for all $c \in \min \left(u^{-1}\right)$. Moreover $\left|u_{1}\right|=\left|u_{2}\right|=|u|$. By Levi's Lemma, there exist traces $x, y_{1}, y_{2}$ and $z$ with $u_{1} b=x y_{1}, u_{2}=y_{2} z, u a=x y_{2}$ and $u^{-1}=y_{1} z$. Assume that $y_{2} \neq \varepsilon$. Since $\max \left(y_{2}\right) \subseteq \max (u a)=\{a\}$ we get $\max \left(y_{2}\right)=\{a\}$. Since $(a, c) \in D$ for all $c \in \min \left(y_{1}\right) \subseteq \min \left(u^{-1}\right)$ and $y_{1} I y_{2}$ it follows $y_{1}=\varepsilon$. But then $|u|=\left|u^{-1}\right|=|z|<\left|y_{2} z\right|=\left|u_{2}\right|$ leads to a contradiction. Hence, we must have $y_{2}=\varepsilon$. Thus $|u|=\left|u^{-1}\right|=\left|y_{1} z\right|=\left|y_{1}\right|+|z|=\left|y_{1}\right|+\left|u_{2}\right|=$ $\left|y_{1}\right|+|u|$ implies $y_{1}=\varepsilon$. Therefore we get $u a=u_{1} b$ and $u^{-1}=u_{2}$. Finally, since $\max (u a)=\{a\}$ we must have $a=b$ and $u=u_{1}$.

Lemma 16. Let $w \in \mathbb{M}\left(\Sigma^{ \pm 1}, I\right)$ be R-irreducible and $a \in \Sigma^{ \pm 1}$. Then the following three conditions are equivalent:

(1) There exists $x \in \mathbb{M}\left(\Sigma^{ \pm 1}, I\right)$ with $w=x^{-1}$ in $\mathbb{G}(\Sigma, I)$.

(2) There exists $x \in \mathbb{M}\left(\Sigma^{ \pm 1}, I\right)$ with $w=\operatorname{xax}^{-1}$ in $\mathbb{M}\left(\Sigma^{ \pm 1}, I\right)$.

(3) $w$ is a double a-cone.

Proof. Direction " $(2) \Rightarrow(1)$ " is trivially true and " $(2) \Leftrightarrow(3)$ ” is just the definition of a double $a$-cone. For " $(1) \Rightarrow(2)$ " assume that $w=x^{-1}$ in $\mathbb{G}(\Sigma, I)$. Since $w \in \operatorname{IRR}(R)$, we have $x_{a x}{ }^{-1} \stackrel{*}{\rightarrow}_{R} w$. W.l.o.g., $x \in \operatorname{IRR}(R)$. Let $n \geq 0$ such that $\operatorname{xax}^{-1} \rightarrow_{R}^{n} w$. We prove (2) by induction on $n$.

For $n=0$ we have $w=x a x^{-1}$ in $\mathbb{M}\left(\Sigma^{ \pm 1}, I\right)$. So assume $n>0$. If $a \in \max (x)$ we have $x=y a$ for some $y \in \mathbb{M}\left(\Sigma^{ \pm 1}, I\right)$ and hence $x a x^{-1}=y a a a^{-1} y^{-1} \rightarrow_{R} y a y^{-1}$. Since $R$ is confluent, we have $y a y^{-1} \stackrel{*}{\rightarrow}_{R} w$ and since each rewriting rule from $R$ reduces the length of a trace by 2 it follows that $y_{a y}{ }^{-1} \rightarrow_{R}^{n-1} w$. Hence, by induction, there exists a trace $v$ with $w=v a v^{-1}$ in $\mathbb{M}\left(\Sigma^{ \pm 1}, I\right)$. The case where $a^{-1} \in \max (x)$ is analogous to the previous case. If there exists $b \in \max (x)$ with $(a, b) \in I$ we can infer that $x=y b$ for some trace $y$ and $x a x^{-1}=y b a b^{-1} y^{-1}=y a b b^{-1} y^{-1} \rightarrow_{R} y a y^{-1}$. As for the previous cases we obtain inductively $w=v a v^{-1}$ in $\mathbb{M}\left(\Sigma^{ \pm 1}, I\right)$ for some trace $v$. Finally, if $\max (x) \cap\left(\left\{a, a^{-1}\right\} \cup I(a)\right)=\emptyset$, then $x a x^{-1}$ is a double $a$-cone by Lemma 14 and hence $R$-irreducible, which contradicts $n>0$.

Lemma 17. Let $w_{a}, v_{a} \in \mathbb{M}\left(\Sigma^{ \pm 1}, I\right)$ ( $\left.a \in \Sigma\right)$ be R-irreducible such that $w_{a}=$ $v_{a} a v_{a}^{-1}$ in $\mathbb{M}\left(\Sigma^{ \pm 1}, I\right)$ for all $a \in \Sigma$ (thus, every $w_{a}$ is a double a-cone). If there is a trace $x \in \mathbb{M}\left(\Sigma^{ \pm 1}, I\right)$ with $x a x^{-1}=w_{a}$ in $\mathbb{G}(\Sigma, I)$ for all $a \in \Sigma$, then $s=\bigsqcup_{a \in \Sigma} v_{a}$ exists and sas ${ }^{-1}=w_{a}$ in $\mathbb{G}(\Sigma, I)$ for all $a \in \Sigma$. 
Proof. Assume that a trace $x \in \mathbb{M}\left(\Sigma^{ \pm 1}, I\right)$ exists with $x a x^{-1}=w_{a}$ in $\mathbb{G}(\Sigma, I)$ for all $a \in \Sigma$. We can assume w.l.o.g. that $x \in \operatorname{IRR}(R)$. First, write $x$ as $x=x_{a} a^{n_{a}}$ with $n_{a} \in \mathbb{Z}$ and $\left|n_{a}\right|$ maximal for every $a \in \Sigma$. Then $a, a^{-1} \notin \max \left(x_{a}\right)$ and $x_{a}$ is uniquely determined by the cancellativity of $\mathbb{M}\left(\Sigma^{ \pm 1}, I\right)$. Next we write $x_{a}$ as $x_{a}=t_{a} u_{a}$ with $u_{a} I a$ and $\left|u_{a}\right|$ maximal. This decomposition is unique by Lemma 8. We get

$$
x a x^{-1}=t_{a} u_{a} a^{n_{a}} a a^{-n_{a}} u_{a}^{-1} t_{a}^{-1} \stackrel{*}{\rightarrow}_{R} t_{a} u_{a} a u_{a}^{-1} t_{a}^{-1} \stackrel{*}{\rightarrow}_{R} t_{a} a t_{a}^{-1} \stackrel{*}{\rightarrow}_{R} w_{a}=v_{a} a v_{a}^{-1} .
$$

From the choice of $n_{a}$ and $u_{a}$ it follows that $\max \left(t_{a}\right) \cap\left(\left\{a, a^{-1}\right\} \cup I(a)\right)=\emptyset$. This implies that $t_{a} a t_{a}^{-1} \in \operatorname{IRR}(R)$. Hence $t_{a} a t_{a}^{-1}=v_{a} a v_{a}^{-1}$ in $\mathbb{M}\left(\Sigma^{ \pm 1}, I\right)$ and by Lemma 15 it follows that $t_{a}=v_{a}$. So for all $a \in \Sigma$ it holds that $v_{a} \preceq x$ and therefore $s=\bigsqcup_{a \in \Sigma} v_{a}$ exists.

Now we infer that $\operatorname{sas}^{-1}=w_{a}$ in $\mathbb{G}(\Sigma, I)$ for all $a \in \Sigma$. Since $v_{a} \preceq x$ for all $a \in \Sigma$, there is some trace $y$ such that $x=s y$ in $\mathbb{M}\left(\Sigma^{ \pm 1}, I\right)$. We can write $s=v_{a} r_{a}$ for all $a \in \Sigma$. Let $z_{a}=r_{a} y$ and hence $x=v_{a} r_{a} y=v_{a} z_{a}$. As a suffix of the $R$ irreducible trace $x, z_{a}$ is $R$-irreducible as well. By assumption we have

$$
\forall a \in \Sigma: v_{a} a v_{a}^{-1}=w_{a}=x a x^{-1}=v_{a} z_{a} a z_{a}^{-1} v_{a}^{-1}
$$

in $\mathbb{G}(\Sigma, I)$ and hence, by cancelling $v_{a}$ and $v_{a}^{-1}$,

$$
\forall a \in \Sigma: a=z_{a} a z_{a}^{-1}
$$

in $\mathbb{G}(\Sigma, I)$. Since $a$ as a single symbol is $R$-irreducible, this means that

$$
\forall a \in \Sigma: z_{a} a z_{a}^{-1} \rightarrow_{R}^{*} a .
$$

We prove by induction on $\left|z_{a}\right|$ that $\operatorname{alph}\left(z_{a}\right) \subseteq I(a) \cup\left\{a, a^{-1}\right\}$. The case $z_{a}=\varepsilon$ is clear. Now assume that $z_{a} \neq \varepsilon$. If every maximal symbol in $z_{a}$ belongs to $\Sigma^{ \pm 1} \backslash(I(a) \cup$ $\left\{a, a^{-1}\right\}$ ), then $z_{a} a z_{a}^{-1} \in \operatorname{IRR}(R)$ (recall that $z_{a} \in \operatorname{IRR}(R)$ ), which contradicts (1). Hence, let $z_{a}=z_{a}^{\prime} b$ with $b \in I(a) \cup\left\{a, a^{-1}\right\}$. We get $z_{a} a z_{a}^{-1} \rightarrow_{R} z_{a}^{\prime} a z_{a}^{\prime-1} \rightarrow_{R}^{*} a$. By induction, it follows that $\operatorname{alph}\left(z_{a}^{\prime}\right) \subseteq I(a) \cup\left\{a, a^{-1}\right\}$. Hence, the same is true for $z_{a}$ and therefore for the prefix $r_{a}$ of $z_{a}$ as well. But this implies $s a s^{-1}=v_{a} r_{a} a r_{a}^{-1} v_{a}^{-1}=$ $v_{a} a v_{a}^{-1}=w_{a}$ in $\mathbb{G}(\Sigma, I)$.

\section{Restricted simultaneous compressed conjugacy}

Based on our results on double $a$-cones from the previous section, we will prove Theorem 2 in this section. First, we have to prove the following lemma:

Lemma 18. Let $a \in \Sigma^{ \pm 1}$. For a given $\operatorname{SLP} \mathbb{A}$ with $[\operatorname{val}(\mathbb{A})]_{I} \in \operatorname{IRR}(R)$, we can check in polynomial time whether $[\operatorname{val}(\mathbb{A})]_{I}$ is a double a-cone. In case $[\mathrm{val}(\mathbb{A})]_{I}$ is a double a-cone, we can compute in polynomial time an $S L P \mathbb{V}$ over $\Sigma^{ \pm 1}$ with $\operatorname{val}(\mathbb{A})=$ $\operatorname{val}(\mathbb{V}) a \operatorname{val}\left(\mathbb{V}^{-1}\right)$ in $\mathbb{M}\left(\Sigma^{ \pm 1}, I\right)$.

Proof. First we check whether $|\operatorname{val}(\mathbb{A})|$ is odd. If not, then $[\operatorname{val}(\mathbb{A})]_{I}$ cannot be a double $a$-cone. Assume that $|\operatorname{val}(\mathbb{A})|=2 k+1$ for some $k \geq 0$ and let $\operatorname{val}(\mathbb{A})=u_{1} b u_{2}$ with 
$\left|u_{1}\right|=\left|u_{2}\right|=k$. By [13] we can construct SLPs $\mathbb{V}_{1}$ and $\mathbb{V}_{2}$ such that $\operatorname{val}\left(\mathbb{V}_{1}\right)=$ $\operatorname{val}(\mathbb{A})[1: k]=u_{1}$ and $\operatorname{val}\left(\mathbb{V}_{2}\right)=\operatorname{val}(\mathbb{A})[k+2: 2 k+1]=u_{2}$. By Lemma 15, $[\operatorname{val}(\mathbb{A})]_{I}$ is a double $a$-cone if and only if $a=b$ and $\left[\operatorname{val}\left(\mathbb{V}_{1}\right)\right]_{I}=\left[\operatorname{val}\left(\mathbb{V}_{2}^{-1}\right)\right]_{I}$. This can be checked in polynomial time.

Now we are in the position to present a polynomial time algorithm for $\operatorname{RSCCP}(\Sigma, I)$ :

Proof of Theorem 2. Let $\mathbb{A}_{a}(a \in \Sigma)$ be the input SLPs. We have to check whether there exists $x$ such that $\operatorname{val}\left(\mathbb{A}_{a}\right)=x a x^{-1}$ in $\mathbb{G}(\Sigma, I)$ for all $a \in \Sigma$. Since the SLP $\mathbb{A}_{a}$ and an $R$-reduction of $\mathbb{A}_{a}$ represent the same group element in $\mathbb{G}(\Sigma, I)$, Theorem 9 allows us to assume that the input SLPs $\mathbb{A}_{a}(a \in \Sigma)$ represent $R$-irreducible traces.

We first check whether every trace $\left[\operatorname{val}\left(\mathbb{A}_{a}\right)\right]_{I}$ is a double $a$-cone. By Lemma 18 this is possible in polynomial time. If there exists $a \in \Sigma$ such that $\left[\operatorname{val}\left(\mathbb{A}_{a}\right)\right]_{I}$ is not a double $a$-cone, then we can reject by Lemma 16. Otherwise, we can compute (using again Lemma 18) SLPs $\mathbb{V}_{a}(a \in \Sigma)$ such that $\left[\operatorname{val}\left(\mathbb{A}_{a}\right)\right]_{I}=\left[\operatorname{val}\left(\mathbb{V}_{a}\right)\right]_{I} a\left[\operatorname{val}\left(\mathbb{V}_{a}\right)\right]_{I}^{-1}$ in $\mathbb{M}\left(\Sigma^{ \pm 1}, I\right)$. Finally, by Lemma 17 , it suffices to check whether $\bigsqcup_{a \in \Sigma}\left[\operatorname{val}\left(\mathbb{V}_{a}\right)\right]_{I}$ exists, which is possible in polynomial time by Corollary 13 (recall that $|\Sigma|$ is a constant in our consideration). Moreover, if this supremum exists, then we can compute in polynomial time an SLP $\mathbb{S}$ with $[\operatorname{val}(\mathbb{S})]_{I}=\bigsqcup_{a \in \Sigma}\left[\operatorname{val}\left(\mathbb{V}_{a}\right)\right]_{I}$. Then, $\operatorname{val}(\mathbb{S})$ is a solution for our $\operatorname{RSCCP}(\Sigma, I)$-instance.

\section{Computing the core of a compressed trace}

In order to prove Theorem 1 we need some further concepts from [28].

Definition 19. A trace $y$ is called cyclically $R$-irreducible if $y \in \operatorname{IRR}(R)$ and $\min (y) \cap$ $\min \left(y^{-1}\right)=\emptyset$. If for a trace $x$ we have $\mathrm{NF}_{R}(x)=$ uyu $u^{-1}$ in $\mathbb{M}\left(\Sigma^{ \pm 1}, I\right)$ for traces $y, u$ with $y$ cyclically $R$-irreducible, then we call $y$ the core of $x$, core $(x)$ for short.

The trace $y$ in the last definition is uniquely defined [28]. Moreover, note that a trace $t$ is a double $a$-cone if and only if $t \in \operatorname{IRR}(R)$ and core $(t)=a$.

In this section, we will present a polynomial time algorithm for computing an SLP that represents $\operatorname{core}\left([\operatorname{val}(\mathbb{A})]_{I}\right)$ for a given SLP $\mathbb{A}$. For this, we need the following lemmas.

Lemma 20. Let $p, t \in \mathbb{M}\left(\Sigma^{ \pm 1}, I\right)$. If $p \preceq t, p^{-1} \preceq t$ and $t \in \operatorname{IRR}(R)$, then $p=\varepsilon$.

Proof. Suppose for contradiction that

$$
T=\left\{t \in \operatorname{IRR}(R) \mid \exists p \in \mathbb{M}\left(\Sigma^{ \pm 1}, I\right) \backslash\{\varepsilon\}: p \preceq t \wedge p^{-1} \preceq t\right\} \neq \emptyset .
$$

Let $t \in T$ with $|t|$ minimal and $p \in \mathbb{M}\left(\Sigma^{ \pm 1}, I\right)$ such that $p \neq \varepsilon, p \preceq t$, and $p^{-1} \preceq t$. If $|p|=1$ then $p=a$ for some $a \in \Sigma^{ \pm 1}$ and hence $a \preceq t$ and $a^{-1} \preceq t$, a contradiction since $a D a^{-1}$. If $|p|=2$ then $p=a_{1} a_{2}$ for some $a_{1}, a_{2} \in \Sigma^{ \pm 1}$. Since $t$, and therefore $p$ is $R$-irreducible, we have $a_{1} \neq a_{2}^{-1}$. Since $a_{1} \in \min (t)$ and $a_{2}^{-1} \in \min (t)$ we have $a_{1} I a_{2}^{-1}$, i.e., $a_{1} I a_{2}$. Hence, also $a_{2} \in \min (t)$, which contradicts $a_{2}^{-1} \in \min (t)$. So assume that $|p|>2$. Let $a \in \min (p)$. Then $a \in \min (t)$, and there exist traces $y, t^{\prime}$ 
with $t=a t^{\prime}=p^{-1} y$. If $a \notin \min \left(p^{-1}\right)$, then $a \in \min (y)$ and $a I p^{-1}$. But the latter independence contradicts $a^{-1} \in \operatorname{alph}\left(p^{-1}\right)$. Hence $a \in \min \left(p^{-1}\right)$, i.e., $a^{-1} \in \max (p)$. Thus, we can write $p=a q a^{-1}$ and $p^{-1}=a q^{-1} a^{-1}$ with $q \neq \varepsilon$. Since $a q a^{-1}=p \preceq$ $a t^{\prime}, a q^{-1} a^{-1}=p^{-1} \preceq a t^{\prime}$ and $\mathbb{M}\left(\Sigma^{ \pm 1}, I\right)$ is cancellative, we have a $q \preceq t^{\prime}, q^{-1} \preceq t^{\prime}$. Since $q \neq \varepsilon$, we have a contradiction to the fact that $|t|$ is minimal.

Example 21. We take the independence alphabet from Example 6 and consider the trace $x=\left[c^{-1} d^{-1} a^{-1} b a^{-1} c a b d c^{-1} d^{-1} a^{-1} b^{-1} d c a\right]_{I} \in \mathbb{M}\left(\Sigma^{ \pm 1}, I\right)$, whose dependence graph looks as follows:

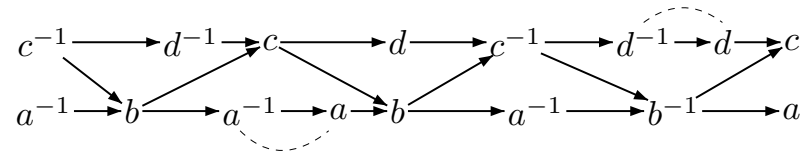

Then the $R$-reduction of $x$ is $\operatorname{NF}_{R}(x)=\left[c^{-1} d^{-1} a^{-1} b c b d c^{-1} a^{-1} b^{-1} c a\right]_{I}$ :

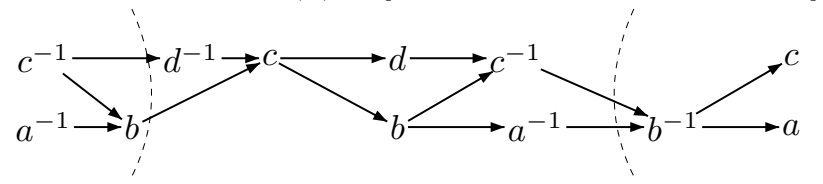

Hence, the core of $x$ is core $(x)=\left[d^{-1} c b d c^{-1} a^{-1}\right]_{I}$ and looks as follows:

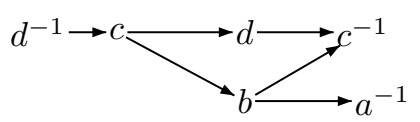

Note that we have $\operatorname{NF}_{R}(x) \sqcap \mathrm{NF}_{R}\left(x^{-1}\right)=c^{-1} a^{-1} b$ and hence

$$
\begin{aligned}
\mathrm{NF}_{R}\left(\left(\operatorname{NF}_{R}(x) \sqcap\right.\right. & \left.\operatorname{NF}_{R}\left(x^{-1}\right)\right)^{-1} \operatorname{NF}_{R}(x)\left(\operatorname{NF}_{R}(x) \sqcap \mathrm{NF}_{R}\left(x^{-1}\right)\right) \\
& =\operatorname{NF}_{R}\left(\left(c^{-1} a^{-1} b\right)^{-1}\left(c^{-1} d^{-1} a^{-1} b c b d c^{-1} a^{-1} b^{-1} c a\right)\left(c^{-1} a^{-1} b\right)\right) \\
& =d^{-1} c b d c^{-1} a^{-1}=\operatorname{core}(x) .
\end{aligned}
$$

This fact holds for every trace, and shall be proven next.

Lemma 22. Let $x \in \operatorname{IRR}(R)$ and $d=x \sqcap x^{-1}$. Then $\operatorname{NF}_{R}\left(d^{-1} x d\right)=\operatorname{core}(x)$.

Proof. Let $d=x \sqcap x^{-1}$. Thus, there are traces $y, z$ such that $d y=x=z^{-1} d^{-1}$ and $\min (y) \cap \min (z)=\emptyset$. By Levi's Lemma it follows that there are traces $u, v_{1}, v_{2}, w$ such that $u v_{1}=d, v_{2} w=y, u v_{2}=z^{-1}, v_{1} w=d^{-1}$, and $v_{1} I v_{2}$. Hence we have $v_{1}^{-1} \preceq d^{-1}$ and $v_{1} \preceq d^{-1}$ and since $x$ is $R$-irreducible, so is $d^{-1}$. We can apply Lemma 20 to infer that $v_{1}=\varepsilon$.

It follows that $u=d, w=d^{-1}$, and thus $x=d y=d v_{2} w=d v_{2} d^{-1}$. Moreover, since $\min \left(v_{2} w\right) \cap \min \left(v_{2}^{-1} u^{-1}\right)=\min (y) \cap \min (z)=\emptyset$, we have $\min \left(v_{2}\right) \cap$ $\min \left(v_{2}^{-1}\right)=\emptyset$. Hence, $v_{2}$ is the core of $x$. Moreover since $x$ (and therefore $v_{2}$ ) is $R$ irreducible, we have $\operatorname{NF}_{R}\left(d^{-1} x d\right)=\operatorname{NF}_{R}\left(d^{-1} d v_{2} d^{-1} d\right)=v_{2}$.

We now easily obtain: 
Corollary 23. Fix an independence alphabet $\left(\Sigma^{ \pm 1}, I\right)$. Then, the following problem can be solved in polynomial time:

INPUT: An SLP $\mathbb{A}$

OUTPUT: An $S L P \mathbb{B}$ with $[\operatorname{val}(\mathbb{B})]_{I}=\operatorname{core}\left([\operatorname{val}(\mathbb{A})]_{I}\right)$

Proof. By Theorem 9 we can assume that $[\operatorname{val}(\mathbb{A})]_{I} \in \operatorname{IRR}(R)$. Then, using Theorem 11 we can compute in polynomial time an SLP $\mathbb{P}$ with $[\operatorname{val}(\mathbb{P})]_{I}=[\operatorname{val}(\mathbb{A})]_{I} \sqcap\left[\operatorname{val}(\mathbb{A})^{-1}\right]_{I}$. By Lemma 22 we have core $\left([\operatorname{val}(\mathbb{A})]_{I}\right)=\mathrm{NF}_{R}\left(\left[\operatorname{val}(\mathbb{P})^{-1} \operatorname{val}(\mathbb{A}) \operatorname{val}(\mathbb{P})\right]_{I}\right)$. Finally, by Theorem 9 we can compute in polynomial time an SLP $\mathbb{B}$ such that $[\operatorname{val}(\mathbb{B})]_{I}=$ $\mathrm{NF}_{R}\left(\left[\operatorname{val}(\mathbb{P})^{-1} \operatorname{val}(\mathbb{A}) \operatorname{val}(\mathbb{P})\right]_{I}\right)$.

\section{A pattern matching algorithm for connected patterns}

Our second tool for proving Theorem 1 is a pattern matching algorithm for compressed traces. For two traces $v$ and $w$ we say that $v$ is a factor of $w$ if there is some trace $u$ such that $u v \preceq w$. We consider the following problem and show that it can be solved in polynomial time if the independence alphabet $(\Sigma, I)$ satisfies certain conditions.

INPUT: An independence alphabet $(\Sigma, I)$ and two SLPs $\mathbb{T}$ and $\mathbb{P}$ over $\Sigma$.

QUESTION: Is $[\operatorname{val}(\mathbb{P})]_{I}$ a factor of $[\operatorname{val}(\mathbb{T})]_{I}$ ?

We write $\operatorname{alph}(\mathbb{T})$ and alph $(\mathbb{P})$ for alph $(\operatorname{val}(\mathbb{T}))$ and alph $(\operatorname{val}(\mathbb{P}))$, respectively. We may assume that $\Sigma=\operatorname{alph}(\mathbb{T})$ and that $\Sigma$ is connected. Otherwise we simply solve several instances of the latter problem separately. Also, we assume in the following that the SLPs $\mathbb{T}=(V, \Sigma, S, P)$ and $\mathbb{P}$ are in Chomsky normal form. Let $\Gamma \subseteq \Sigma$. We denote by $\pi_{\Gamma}$, the homomorphism $\pi_{\Gamma}: \mathbb{M}(\Sigma, I) \rightarrow \mathbb{M}(\Gamma, I \cap(\Gamma \times \Gamma))$ with $\pi_{\Gamma}(a)=a$ for $a \in \Gamma$ and $\pi_{\Gamma}(a)=\varepsilon$ for $a \in \Sigma \backslash \Gamma$. Let $V^{\Gamma}=\left\{X^{\Gamma} \mid X \in V\right\}$ be a disjoint copy of $V$. For each production $p \in P$ define a new production $p^{\Gamma}$ as follows. If $p$ is of the form $X \rightarrow a(a \in \Sigma)$, then let $p^{\Gamma}=\left(X^{\Gamma} \rightarrow a\right)$ in case $a \in \Gamma$ and $p^{\Gamma}=$ $\left(X^{\Gamma} \rightarrow \varepsilon\right)$ otherwise. Moreover, if $p \in P$ is of the form $X \rightarrow Y Z(X, Y, Z \in V)$ define $p^{\Gamma}=\left(X^{\Gamma} \rightarrow Y^{\Gamma} Z^{\Gamma}\right)$. We denote with $\mathbb{T}^{\Gamma}$ the $\operatorname{SLP}\left(V^{\Gamma}, \Gamma, S^{\Gamma}, P^{\Gamma}\right)$ where $P^{\Gamma}=\left\{p^{\Gamma} \mid p \in P\right\}$. Obviously, $\operatorname{val}\left(\mathbb{T}^{\Gamma}\right)=\pi_{\Gamma}(\operatorname{val}(\mathbb{T}))$.

In order to develop a polynomial time algorithm for the problem stated above we need a succinct representation for an occurrence of $\mathbb{P}$ in $\mathbb{T}$. Since $[\operatorname{val}(\mathbb{P})]_{I}$ is a factor of $[\operatorname{val}(\mathbb{T})]_{I}$ iff there is a prefix $u \preceq[\operatorname{val}(\mathbb{T})]_{I}$ such that $u[\operatorname{val}(\mathbb{P})]_{I} \preceq[\operatorname{val}(\mathbb{T})]_{I}$, we will in fact compute prefixes with the latter property and represent a prefix $u$ by its Parikh image $\left(|u|_{a}\right)_{a \in \Sigma}$. Hence we say a sequence $O=\left(O_{a}\right)_{a \in \Sigma} \in \mathbb{N}^{\Sigma}$ is an occurrence of a trace $v$ in a trace $w$ iff there is a prefix $u \preceq w$ such that $u v \preceq w$, and $O=\left(|u|_{a}\right)_{a \in \Sigma}$. For $\Gamma \subseteq \Sigma$ we write $\pi_{\Gamma}(O)$ for the restriction $\left(O_{a}\right)_{a \in \Gamma}$. Furthermore, we say that $O$ is an occurrence of $\mathbb{P}$ in $\mathbb{T}$ if $O$ is an occurrence of $[\operatorname{val}(\mathbb{P})]_{I}$ in $[\operatorname{val}(\mathbb{T})]_{I}$. Note that our definition of an occurrence of $\mathbb{P}$ in $\mathbb{T}$ does not exactly correspond to the intuitive notion of an occurrence as a convex subset of the dependence graph of $[\operatorname{val}(\mathbb{T})]_{I}$. In fact, to a convex subset of the dependence graph of $[\operatorname{val}(\mathbb{T})]_{I}$, which is isomorphic to the dependence graph of $[\operatorname{val}(\mathbb{P})]_{I}$, there might correspond several occurrences $O$, since for an $a \in \Sigma$ that is independent of alph $(\mathbb{P})$ we might have several possibilities for the 
value $O_{a}$. However, if we restrict to letters that are dependent on alph $(\mathbb{P})$, then our definition of an occurrence coincides with the intuitive notion.

Let $X$ be a nonterminal of $\mathbb{T}$ with production $X \rightarrow Y Z$ and let $O$ be an occurrence of $[\operatorname{val}(\mathbb{P})]_{I}$ in $[\operatorname{val}(X)]_{I}$. If there are $a, b \in \operatorname{alph}(\mathbb{P})$ such that $O_{a}<|\operatorname{val}(Y)|_{a}$ and $O_{b}+|\operatorname{val}(\mathbb{P})|_{b}>|\operatorname{val}(Y)|_{b}$, then we say that $O$ is an occurrence of $\mathbb{P}$ at the cut of $X$. We assume w.l.o.g. that $|\operatorname{val}(\mathbb{P})| \geq 2$, otherwise the problem reduces simply to checking whether there occurs a certain letter in $\operatorname{val}(\mathbb{T})$. This assumption implies that $[\operatorname{val}(\mathbb{P})]_{I}$ is a factor of $[\operatorname{val}(\mathbb{T})]_{I}$ if and only if there is a nonterminal $X$ of $\mathbb{T}$ for which there is an occurrence of $\mathbb{P}$ at the cut of $X$.

Example 24. We take the independence alphabet from Example 6 again. Let $X$ be a nonterminal with $\operatorname{val}(X)=a c b c$ ad $c b c a c b c a c b c a c b c a c b \mid c a c b c a c b c a c b c a c b d c$ where 'l' denotes the cut of $X$ and $\operatorname{val}(\mathbb{P})=a c b c a c b c a c b c a c b c a c b c$. Then the occurrences of $\operatorname{val}(\mathbb{P})$ at the cut of $X$ are $(1,1,2,1),(2,2,4,1),(3,3,6,1)$ and $(4,4,8,1)$ where the positions in a tuple correspond to the letters in our alphabet in the order $a, b, c, d$. We will see later how to construct them.

Lemma 25 ([16]). Let $v$ and $w$ be traces over $\Sigma$. A sequence $\left(n_{a}\right)_{a \in \Sigma} \in \mathbb{N}^{\Sigma}$ is an occurrence of $v$ in $w$ if and only if $\left(n_{a}, n_{b}\right)$ is an occurrence of $\pi_{\{a, b\}}(v)$ in $\pi_{\{a, b\}}(w)$ for all $(a, b) \in D$.

An arithmetic progression is a subset of $\mathbb{N}^{\Sigma}$ of the form

$$
\left\{\left(i_{a}\right)_{a \in \Sigma}+k \cdot\left(d_{a}\right)_{a \in \Sigma} \mid 0 \leq k \leq \ell\right\} .
$$

This set can be represented by the triple $\left(\left(i_{a}\right)_{a \in \Sigma},\left(d_{a}\right)_{a \in \Sigma}, \ell\right)$. The descriptional size $\left|\left(\left(i_{a}\right)_{a \in \Sigma},\left(d_{a}\right)_{a \in \Sigma}, \ell\right)\right|$ of the arithmetic progression $\left(\left(i_{a}\right)_{a \in \Sigma},\left(d_{a}\right)_{a \in \Sigma}, \ell\right)$ is $\log _{2}(\ell)+$ $\sum_{a \in \Sigma}\left(\log _{2}\left(i_{a}\right)+\log _{2}\left(d_{a}\right)\right)$. In Example 24, the occurrences of val $(\mathbb{P})$ at the cut of $X$ form the arithmetic progression $((1,1,2,1),(1,1,2,0), 3)$.

We will use the last lemma in order to compute the occurrences of $\mathbb{P}$ in $\mathbb{T}$ in form of a family of arithmetic progressions. To this aim, we follow a similar approach as Genest and Muscholl for message sequence charts [12]. In particular Lemma 26 below was inspired by [12, Proposition 1].

Throughout the rest of this section we make the following assumption:

$\operatorname{alph}(\mathbb{P})$ is connected and $\{a, b\} \cap \operatorname{alph}(\mathbb{P}) \neq \emptyset$ for all $(a, b) \in D$ with $a \neq b$.

Let $X$ be a nonterminal of $\mathbb{T}$ and let $O$ be an occurrence of $\mathbb{P}$ at the cut of $X$. Since the pattern is connected there must be some $a, b \in \Sigma$ with $(a, b) \in D$ such that $\pi_{\{a, b\}}(O)$ is at the cut of $X^{\{a, b\}}$. We will therefore compute occurrences of $\pi_{\{a, b\}}(\operatorname{val}(\mathbb{P}))$ at the cut of $X^{\{a, b\}}$. It is well known that the occurrences of $\pi_{\{a, b\}}(\operatorname{val}(\mathbb{P}))$ at the cut of $X^{\{a, b\}}$ form an arithmetic progression $\left(\left(i_{a}, i_{b}\right),\left(d_{a}, d_{b}\right), \ell\right)$ and that $\pi_{\{a, b\}}(\operatorname{val}(\mathbb{P}))$ is of the form $u^{n} v$ for some $n \geq \ell$ and strings $u, v \in\{a, b\}^{*}$ with $v \preceq u,|u|_{a}=d_{a}$ and $|u|_{b}=$ $d_{b}$. Moreover, the arithmetic progression $\left(\left(i_{a}, i_{b}\right),\left(d_{a}, d_{b}\right), \ell\right)$ can be computed in time $|\mathbb{T}|^{2}|\mathbb{P}|\left(\right.$ see $\left.[15]^{2}\right)$. Now suppose we have computed the occurrences of $\pi_{\{a, b\}}(\operatorname{val}(\mathbb{P}))$

\footnotetext{
${ }^{2}$ In fact, in [15] it was shown that the arithmetic progression $\left(i_{a}+i_{b}, d_{a}+d_{b}, \ell\right)$ can be computed in polynomial time. Observe that from this the arithmetic progression $\left(\left(i_{a}, i_{b}\right),\left(d_{a}, d_{b}\right), \ell\right)$ can easily be computed.
} 
at the cut of $X^{\{a, b\}}$ in form of an arithmetic progression. The problem now is how to find (for the possibly exponentially many occurrences in the arithmetic progression) matching occurrences of projections onto all other pairs in $D$.

The following lemma states that either there is a pair $(a, b) \in D$ such that the projection onto $\{a, b\}$ is the first or the last element of an arithmetic progression, or all projections lie at the cut of the same nonterminal.

Lemma 26. Let $X$ be a nonterminal of $\mathbb{T}$ and let $O$ be an occurrence of $\mathbb{P}$ at the cut of $X$. Then either

(i) $\pi_{\{a, b\}}(O)$ is at the cut of $X^{\{a, b\}}$ for all $(a, b) \in D$ with $a \neq b$, or

(ii) there are $a, b \in \operatorname{alph}(\mathbb{P})$ with $(a, b) \in D$ such that $\pi_{\{a, b\}}(O)$ is the first or last element of the arithmetic progression of occurrences of $\pi_{\{a, b\}}(\mathrm{val}(\mathbb{P}))$ at the cut of $X^{\{a, b\}}$.

Proof. Let $X \rightarrow Y Z$ be a production of $\mathbb{T}$. Clearly, by our general assumption (2) it suffices to show that either (ii) holds, or $O_{a}<|\operatorname{val}(Y)|_{a}<O_{a}+|\operatorname{val}(\mathbb{P})|_{a}$ for all $a \in \operatorname{alph}(\mathbb{P})$. We show this assertion by induction on $\mid$ alph $(\mathbb{P}) \mid$. If alph $(\mathbb{P})$ is a singleton, then it is trivially true.

Next, we consider the case $|\operatorname{alph}(\mathbb{P})|=2$. So let $\{a, b\}=\operatorname{alph}(\mathbb{P})$ and hence $(a, b) \in D$ by (2). Assume that (ii) does not hold. Consider the arithmetic progression $\left(\left(i_{a}, i_{b}\right),\left(d_{a}, d_{b}\right), \ell\right)$ of occurrences of $\operatorname{val}(\mathbb{P})$ at the cut of $X^{\{a, b\}}$. Then $\operatorname{val}(\mathbb{P})$ is of the form $u^{n} v$ for some $n \geq \ell$ and strings $u, v \in\{a, b\}^{*}$ with $v \preceq u,|u|_{a}=d_{a}$ and $|u|_{b}=d_{b}$. We conclude that $d_{a}, d_{b}>0$ as otherwise $|\operatorname{alph}(\mathbb{P})| \leq 1$. Suppose for contradiction that $i_{a}+\ell d_{a}>|\operatorname{val}(Y)|_{a}$. Since no prefix $w$ of $\pi_{\{a, b\}}(\operatorname{val}(X))$ can satisfy $|w|_{a}<|\operatorname{val}(Y)|_{a}$ and $|w|_{b}>|\operatorname{val}(Y)|_{b}$ we conclude $i_{b}+\ell d_{b} \geq|\operatorname{val}(Y)|_{b}$. But then the occurrence $\left(i_{a}+\ell d_{a}, i_{b}+\ell d_{b}\right)$ is not at the cut of $X^{\{a, b\}}$, which is a contradiction. Hence $i_{a}+\ell d_{a} \leq|\operatorname{val}(Y)|_{a}$ and by symmetry $i_{b}+\ell d_{b} \leq|\operatorname{val}(Y)|_{b}$. Similarly, since $\left(i_{a}, i_{b}\right)$ is an occurrences of $\operatorname{val}(\mathbb{P})$ at the cut of $X^{\{a, b\}}$, we get $|\operatorname{val}(Y)|_{a} \leq i_{a}+|\operatorname{val}(\mathbb{P})|_{a}$ and $|\operatorname{val}(Y)|_{b} \leq i_{b}+|\operatorname{val}(\mathbb{P})|_{b}$. As $\pi_{\{a, b\}}(O)$ is neither the first nor the last element of the arithmetic progression we have $O_{a}=i_{a}+k d_{a}$ and $O_{b}=i_{b}+k d_{b}$ for some $0<k<\ell$ and hence $O_{a}<|\operatorname{val}(Y)|_{a}<O_{a}+|\operatorname{val}(\mathbb{P})|_{a}$ and $O_{b}<|\operatorname{val}(Y)|_{b}<O_{b}+|\operatorname{val}(\mathbb{P})|_{b}$ as required.

Now, suppose that $|\operatorname{alph}(\mathbb{P})| \geq 3$. Since $O$ is an occurrence at the cut of $X$, there are $a, b \in \operatorname{alph}(\mathbb{P})$ such that $O_{a}<|\operatorname{val}(Y)|_{a}$ and $O_{b}+|\operatorname{val}(\mathbb{P})|_{b}>|\operatorname{val}(Y)|_{b}$. We may assume that $(a, b) \in D$. Indeed, if $O_{a}+|\operatorname{val}(\mathbb{P})|_{a}>|\operatorname{val}(Y)|_{a}$ choose $a=b$. Otherwise, since alph $(\mathbb{P})$ is connected there is a dependence path between $a$ and $b$. Since $O_{a}+|\operatorname{val}(\mathbb{P})|_{a} \leq|\operatorname{val}(Y)|_{a}$, there must be an edge $\left(a^{\prime}, b^{\prime}\right) \in D$ on this path such that $a^{\prime}, b^{\prime} \in \operatorname{alph}(\mathbb{P}), O_{a^{\prime}}+|\operatorname{val}(\mathbb{P})|_{a^{\prime}} \leq|\operatorname{val}(Y)|_{a^{\prime}}\left(\right.$ and hence $\left.O_{a^{\prime}}<|\operatorname{val}(Y)|_{a^{\prime}}\right)$, and $O_{b^{\prime}}+|\operatorname{val}(\mathbb{P})|_{b^{\prime}}>|\operatorname{val}(Y)|_{b^{\prime}}$.

Next consider a spanning tree of $(\operatorname{alph}(\mathbb{P}), D \cap$ alph $(\mathbb{P}) \times \operatorname{alph}(\mathbb{P}))$ which contains the edge $(a, b)$ (in case $a \neq b$ ). Let $c \notin\{a, b\}$ be a leaf of this spanning tree (it exists since $|\operatorname{alph}(\mathbb{P})| \geq 3$ ). Obviously, $\Delta=\operatorname{alph}(\mathbb{P}) \backslash\{c\}$ is connected and $\pi_{\Delta}(O)$ is at the cut of $X^{\Delta}$. Thus we can apply the induction hypothesis. Assume again that (ii) does not hold. Applying the induction hypothesis to $\pi_{\Delta}(\operatorname{val}(\mathbb{P}))$ and $X^{\Delta}$ we get $O_{a}<$ $|\operatorname{val}(Y)|_{a}<O_{a}+|\operatorname{val}(\mathbb{P})|_{a}$ for all $a \in \Delta$. In particular, $O_{d}<|\operatorname{val}(Y)|_{d}<O_{d}+$ $|\operatorname{val}(\mathbb{P})|_{d}$ for some $d \in \Delta$ with $(c, d) \in D$. Hence, $\pi_{\{d, c\}}(O)$ is at the cut of $X^{\{d, c\}}$. 
Thus, applying the induction hypothesis also to $\pi_{\{d, c\}}(\operatorname{val}(\mathbb{P}))$ and $X^{\{d, c\}}$ we get $O_{c}<$ $|\operatorname{val}(Y)|_{c}<O_{c}+|\operatorname{val}(\mathbb{P})|_{c}$.

The last lemma motivates that we partition the set of occurrences into two sets. Let $O$ be an occurrence of $\mathbb{P}$ in $\mathbb{T}$ at the cut of $X$. We call $O$ single (for $X$ ) if there are $a, b \in \operatorname{alph}(\mathbb{P})$ with $(a, b) \in D$ such that the projection $\pi_{\{a, b\}}(O)$ is the first or the last element of the arithmetic progression of occurrences of $\pi_{\{a, b\}}(\mathrm{val}(\mathbb{P}))$ at the cut of $X^{\{a, b\}}$. Otherwise, we call $O$ periodic (for $X$ ). By Lemma 26, if $O$ is periodic, then $\pi_{\{a, b\}}(O)$ is an element of the arithmetic progression of occurrences of $\operatorname{val}\left(\mathbb{P}^{\{a, b\}}\right)$ at the cut of $X^{\{a, b\}}$ for all $(a, b) \in D$ (but neither the first nor the last element, if $a, b \in \operatorname{alph}(\mathbb{P}))$. The next proposition shows that we can decide in polynomial time whether there are single occurrences of $\mathbb{P}$ in $\mathbb{T}$.

Proposition 27. Given $a, b \in \operatorname{alph}(\mathbb{P})$ with $(a, b) \in D$, a nonterminal $X$ of $\mathbb{T}$ and an occurrence $\left(O_{a}, O_{b}\right)$ of $\pi_{\{a, b\}}(\operatorname{val}(\mathbb{P}))$ at the cut of $X^{\{a, b\}}$, we can decide in time $(|\mathbb{T}|+|\mathbb{P}|)^{O(1)}$ whether this occurrence is a projection of an occurrence of $\mathbb{P}$ at the cut of $X$.

Proof. Let $a_{1}, \ldots, a_{n}$ be an enumeration of $\Sigma$ such that $a=a_{1}, b=a_{2}$ and $D\left(a_{i}\right) \cap$ $\left\{a_{1}, \ldots, a_{i-1}\right\} \neq \emptyset$ for all $2 \leq i \leq n$. Moreover, we require that the elements of alph $(\mathbb{P})$ appear at the beginning of our enumeration, i.e., are the elements $a_{1}, \ldots, a_{j}$ for some $j \leq n$. This can be assumed since $\Sigma$ and alph $(\mathbb{P})$ are connected. We iterate over $3 \leq i \leq n$ and compute, if possible, an integer $O_{a_{i}}$ such that $\left(O_{a_{1}}, \ldots, O_{a_{i}}\right)$ is an occurrence of $\pi_{\left\{a_{1}, \ldots, a_{i}\right\}}(\operatorname{val}(\mathbb{P}))$ in $\pi_{\left\{a_{1}, \ldots, a_{i}\right\}}(\operatorname{val}(X))$.

So let $i \geq 3, d=a_{i}$, and $\Delta=\left\{a_{1}, \ldots, a_{i-1}\right\}$. By our general assumption (2) we can choose some $c \in \Delta \cap \operatorname{alph}(\mathbb{P})$ such that $(c, d) \in D$. Let us further assume that we have already constructed an occurrence $\left(O_{a_{1}}, \ldots, O_{a_{i-1}}\right)$ of $\pi_{\Delta}(\operatorname{val}(\mathbb{P}))$ in $\pi_{\Delta}(\operatorname{val}(X))$. First, we compute the number $k \geq 0$ such that $d^{k} c$ is a prefix of $\pi_{\{c, d\}}(\operatorname{val}(\mathbb{P}))$. Then, we compute $O_{d}$ such that there is a prefix $w d^{k} c$ of $\pi_{c, d}(\operatorname{val}(X))$ for some $w \in\{c, d\}^{*}$ with $|w|_{c}=O_{c},|w|_{d}=O_{d}$. If such a prefix does not exist, then there is no occurrence $\left(O_{a_{1}}, \ldots, O_{a_{i-1}}, O_{d}\right)$ of $\pi_{\Delta \cup\{d\}}(\operatorname{val}(\mathbb{P}))$ in $\pi_{\Delta \cup\{d\}}(\operatorname{val}(X))$. On the other hand, observe that if there is such an occurrence $\left(O_{a_{1}}, \ldots, O_{a_{i-1}}, O_{d}\right)$, then $O_{d}=|w|_{d}$. Last, using [15] we check in polynomial time for all $e \in D(d) \cap \Delta$ whether $\left(O_{e}, O_{d}\right)$ is an occurrence of $\pi_{\{d, e\}}(\operatorname{val}(\mathbb{P}))$ in $\pi_{\{d, e\}} \operatorname{val}(X)$. By Lemma 25, the latter holds if and only if $\left(O_{a_{1}}, \ldots, O_{a_{i-1}}, O_{d}\right)$ is an occurrence of $\pi_{\Delta \cup\{d\}}(\operatorname{val}(\mathbb{P}))$ in $\pi_{\Delta \cup\left\{a_{i}\right\}}(\operatorname{val}(X))$.

It remains to show that for every nonterminal $X$ of $\mathbb{T}$ we can compute the periodic occurrences. To this aim we define the amalgamation of arithmetic progressions. Let $\Gamma, \Gamma^{\prime} \subseteq \Sigma$ such that $\Gamma \cap \Gamma^{\prime} \neq \emptyset$. Consider two arithmetic progressions

$$
p=\left(\left(i_{a}\right)_{a \in \Gamma},\left(d_{a}\right)_{a \in \Gamma}, \ell\right), \quad p^{\prime}=\left(\left(i_{a}^{\prime}\right)_{a \in \Gamma^{\prime}},\left(d_{a}^{\prime}\right)_{a \in \Gamma^{\prime}}, \ell^{\prime}\right) .
$$

The amalgamation of $p$ and $p^{\prime}$ is

$$
p \otimes p^{\prime}=\left\{v=\left(v_{a}\right)_{a \in \Gamma \cup \Gamma^{\prime}} \mid \pi_{\Gamma}(v) \in p \text { and } \pi_{\Gamma^{\prime}}(v) \in p^{\prime}\right\} .
$$


Example 28. We continue Example 24 and show how to compute occurrences at the cut. First we consider the projections of $\mathbb{P}$ and $X$ :

$$
\begin{array}{ll}
\pi_{\{a, b\}}(\operatorname{val}(\mathbb{P}))=(a b)^{5} & \operatorname{val}\left(X^{\{a, b\}}\right)=(a b)^{6} \mid(a b)^{4} \\
\pi_{\{b, c\}}(\operatorname{val}(\mathbb{P}))=(c b c)^{5} & \operatorname{val}\left(X^{\{b, c\}}\right)=(c b c)^{5} c b \mid c(c b c)^{4} \\
\pi_{\{c, d\}}(\operatorname{val}(\mathbb{P}))=c^{10} & \operatorname{val}\left(X^{\{c, d\}}\right)=c^{2} d c^{9} \mid c^{8} d c
\end{array}
$$

For the projections we find the arithmetic progressions $p_{a b}, p_{b c}, p_{c d}$ of occurrences at the cut:

$$
\begin{aligned}
& \text { occurrences of } \pi_{\{a, b\}}(\operatorname{val}(\mathbb{P})) \text { at the cut of } X^{\{a, b\}}: p_{a b}=((2,2),(1,1), 3) \\
& \text { occurrences of } \pi_{\{b, c\}}(\operatorname{val}(\mathbb{P})) \text { at the cut of } X^{\{b, c\}}: p_{b c}=((1,2),(1,2), 4) \\
& \text { occurrences of } \pi_{\{c, d\}}(\operatorname{val}(\mathbb{P})) \text { at the cut of } X^{\{c, d\}}: p_{c d}=((2,1),(1,0), 7) .
\end{aligned}
$$

Note that in $p_{a b}$ the first component corresponds to $a$ and the second to $b$ whereas in $p_{b c}$ the first component corresponds to $b$ and the second to $c$. We amalgamate the arithmetic progressions and obtain $p_{a b c}=p_{a b} \otimes p_{b c}=((2,2,4),(1,1,2), 3)$. If we again amalgamate we obtain $p_{a b c d}=p_{a b c} \otimes p_{c d}=((2,2,4,1),(1,1,2,0), 2)$. This way we found occurrences $(2,2,4,1),(3,3,6,1)$ and $(4,4,8,1)$ of $\mathbb{P}$ at the cut of $X$. Observe that there is a fourth occurrence $(1,1,2,1)$ that we did not find this way which is single.

Lemma 29. Let $\Gamma, \Gamma^{\prime} \subseteq \Sigma$ with $\Gamma \cap \Gamma^{\prime} \neq \emptyset$, and let $p=\left(\left(i_{a}\right)_{a \in \Gamma},\left(d_{a}\right)_{a \in \Gamma}, \ell\right)$ and $p^{\prime}=\left(\left(i_{a}^{\prime}\right)_{a \in \Gamma^{\prime}},\left(d_{a}^{\prime}\right)_{a \in \Gamma^{\prime}}, \ell^{\prime}\right)$ be two arithmetic progressions. Then $p \otimes p^{\prime}$ is an arithmetic progression which can be computed in time $\left(|p|+\left|p^{\prime}\right|\right)^{O(1)}$.

Proof. We need to solve the system of linear equations

$$
\left[i_{b}+d_{b} \cdot x=i_{b}^{\prime}+d_{b}^{\prime} \cdot y\right]_{b \in \Gamma \cap \Gamma^{\prime}}
$$

for integers $x$ and $y$ under the constraint

$$
0 \leq x \leq \ell \text { and } 0 \leq y \leq \ell^{\prime}
$$

Let us fix an $a \in \Gamma \cap \Gamma^{\prime}$. First we solve the single equation

$$
i_{a}+d_{a} \cdot x=i_{a}^{\prime}+d_{a}^{\prime} \cdot y .
$$

for non-negative integers $x$ and $y$. The solutions are given by the least solution plus a mutliple of the least common multiple of $d_{a}$ and $d_{a}^{\prime}$. We start by computing $g=$ $\operatorname{gcd}\left(d_{a}, d_{a}^{\prime}\right)$. If $i_{a} \neq i_{a}^{\prime} \bmod g$, then there is no solution for equation (5) and hence $p \otimes p^{\prime}=\emptyset$. In this case we stop. Otherwise, we compute the least solution $s_{a} \geq$ $\max \left(i_{a}, i_{a}^{\prime}\right)$ of the simultaneous congruences

$$
\begin{aligned}
& z=i_{a} \quad \bmod d_{a}, \\
& z=i_{a}^{\prime} \quad \bmod d_{a}^{\prime} .
\end{aligned}
$$


This can be accomplished with $\left(\log \left(d_{a}\right)+\log \left(d_{a}^{\prime}\right)\right)^{2}$ many bit operations; see e.g. [1]. Let $k=\left(s_{a}-i_{a}\right) / d_{a} \geq 0$ and $k^{\prime}=\left(s_{a}-i_{a}^{\prime}\right) / d_{a}^{\prime} \geq 0$. Now, the non-negative solutions of equation (5) are given by

$$
(x, y)=\left(k+\frac{d_{a}^{\prime}}{g} \cdot t, k^{\prime}+\frac{d_{a}}{g} \cdot t\right) \text { for all } t \geq 0 .
$$

If $\left|\Gamma \cap \Gamma^{\prime}\right|=1$ we adapt the range for $t$ such that the constraint (4) is satisfied and we are done.

Otherwise, (3) is a system of at least 2 linear equations in 2 variables. Hence (3) has at least 2 (and then infinitely many) solutions iff any two equations are linearly dependent over $\mathbb{Q}$, i.e. for all $b \in \Gamma \cap \Gamma^{\prime}$ the following holds:

$$
\exists k_{b} \in \mathbb{Q}: d_{a}=k_{b} \cdot d_{b}, d_{b}^{\prime}=k_{b} \cdot d_{a}^{\prime} \text { and } i_{a}^{\prime}-i_{a}=k_{b} \cdot\left(i_{b}^{\prime}-i_{b}\right)
$$

In this case all solutions of equation (5) are solutions of equation (3). Thus we can test condition (7) for all $b \in \Gamma \cap \Gamma^{\prime}$ and in case it holds it only remains to adapt the range for $t$ such that the constraint (4) is satisfied. Otherwise there is at most one solution and we can fix $b \in \Gamma \cap \Gamma^{\prime}$ such that (7) does not hold. We plug the solution (6) into $i_{b}+d_{b} \cdot x=i_{b}^{\prime}+d_{b}^{\prime} \cdot y$ and obtain

$$
i_{b}+\left(k+\frac{d_{a}^{\prime}}{g} \cdot t\right) \cdot d_{b}=i_{b}^{\prime}+\left(k^{\prime}+\frac{d_{a}}{g} \cdot t\right) \cdot d_{b}^{\prime} .
$$

We can solve this for $t$ (if possible) and test whether this gives rise to a solution for (3) under the constraint (4).

Proposition 30. Let $X$ be a nonterminal of $\mathbb{T}$. The periodic occurrences of $\mathbb{P}$ at the cut of $X$ form an arithmetic progression which can be computed in time $(|\mathbb{T}|+|\mathbb{P}|)^{O(1)}$.

Proof. As in the proof of Proposition 27 let $a_{1}, \ldots, a_{n}$ be an enumeration of $\Sigma$ such that $\left\{a_{1}, \ldots, a_{i-1}\right\} \cap D\left(a_{i}\right) \neq \emptyset$ for all $2 \leq i \leq n$ and the elements of alph $(\mathbb{P})$ appear at the beginning of the enumeration. We iterate over $1 \leq i \leq n$ and compute the arithmetic progressions of the periodic occurrences of $\pi_{\left\{a_{1}, \ldots, a_{i}\right\}}(\operatorname{val}(\mathbb{P}))$ at the cut of $X^{\left\{a_{1}, \ldots, a_{i}\right\}}$. For $i=1$ this is easy.

So let $i \geq 2$, let $a=a_{i}$ and let $\Delta=\left\{a_{1}, \ldots, a_{i-1}\right\}$. Assume that the periodic occurrences of $\pi_{\Delta}(\operatorname{val}(\mathbb{P}))$ at the cut of $X^{\Delta}$ are given by the arithmetic progression $p=\left(\left(i_{c}\right)_{c \in \Delta},\left(d_{c}\right)_{c \in \Delta}, \ell\right)$. For all $b \in D(a) \cap \Delta$ let

$$
p^{\{a, b\}}=\left(\left(i_{a}^{\{a, b\}}, i_{b}^{\{a, b\}}\right),\left(d_{a}^{\{a, b\}}, d_{b}^{\{a, b\}}\right), n^{\{a, b\}}\right)
$$

be the occurrences of $\pi_{\{a, b\}}(\operatorname{val}(\mathbb{P}))$ at the cut of $X^{\{a, b\}}$ (without the first and the last occurrence if $a, b \in \operatorname{alph}(\mathbb{P})$ ). Recall that we assume that $\{c, d\} \cap$ alph $(\mathbb{P}) \neq \emptyset$ for all $c, d \in \Sigma$ with $(c, d) \in D$ and $c \neq d$. Hence, by Lemma $25, O$ is a periodic occurrence of $\pi_{\left\{a_{1}, \ldots, a_{i}\right\}}(\operatorname{val}(\mathbb{P}))$ at the cut of $X^{\left\{a_{1}, \ldots, a_{i}\right\}}$ if and only if $\pi_{\Delta}(O) \in p$ and $\left(O_{a}, O_{b}\right) \in$ $p^{\{a, b\}}$ for all $b \in D(a) \cap \Delta$. Hence the periodic occurrences of $\pi_{\left\{a_{1}, \ldots, a_{i}\right\}}(\operatorname{val}(\mathbb{P}))$ at the cut of $X^{\left\{a_{1}, \ldots, a_{i}\right\}}$ are given by

$$
\bigotimes_{b \in D(a) \cap \Delta} p^{\{a, b\}} \otimes p
$$

The result follows now from Lemma 29. 
Summarizing the last section we get the following theorem.

Theorem 31. Given an independence alphabet $(\Sigma, I)$, and two SLPs $\mathbb{P}$ and $\mathbb{T}$ over $\Sigma$ such that $\operatorname{alph}(\mathbb{P})=\operatorname{alph}(\mathbb{T})$, we can decide in polynomial time whether $[\operatorname{val}(\mathbb{P})]_{I}$ is a factor of $[\operatorname{val}(\mathbb{T})]_{I}$.

Proof. Note that our assumption (2) is satsified if alph $(\mathbb{P})=\operatorname{alph}(\mathbb{T})$. Recall that we may assume that alph $(\mathbb{T})$ is connected and that $|\operatorname{val}(\mathbb{P})| \geq 2$.

Let $X$ be a nonterminal of $\mathbb{T}$. Using [15] we compute for each pair $(a, b) \in D$ the arithmetic progression of occurrences of $\pi_{a, b}(\operatorname{val}(\mathbb{P}))$ at the cut of $X^{\{a, b\}}$. By applying Proposition 27 to the first and to the last elements of each of these arithmetic progressions, we compute in polynomial time the single occurrences at the cut of $X$. The periodic occurrences can be computed in polynomial time using Proposition 30 . The result follows now since by definition $[\operatorname{val}(\mathbb{P})]_{I}$ is a factor of $[\operatorname{val}(\mathbb{T})]_{I}$ iff there is a nonterminal $X$ of $\mathbb{T}$ such that there is either a single occurrence of $\mathbb{P}$ at the cut of $X$ or a periodic occurrence of $\mathbb{P}$ at the cut of $X$.

Remark 32 . In the last section we actually proved the theorem above under weaker assumptions: We only need for each connected component $\Sigma_{i}$ of alph $(\mathbb{T})$ that $\Sigma_{i} \cap$ $\operatorname{alph}(\mathbb{P})$ is connected and that $\{a, b\} \cap \operatorname{alph}(\mathbb{P}) \neq \emptyset$ for all $(a, b) \in D \cap\left(\Sigma_{i} \times \Sigma_{i}\right)$ with $a \neq b$.

\section{Compressed conjugacy}

In this section we will prove Theorem 1. For this, we will follow the approach from [16, 29] for non-compressed traces. The following result allows us to transfer the conjugacy problem to a problem on (compressed) traces:

Theorem 33 ([16, 29]). Let $u, v \in \mathbb{M}\left(\Sigma^{ \pm 1}, I\right)$. Then the following are equivalent:

(1) $u$ is conjugated to $v$ in $\mathbb{G}(\Sigma, I)$.

(2) There exists $x \in \mathbb{M}\left(\Sigma^{ \pm 1}, I\right)$ such that $x \operatorname{core}(u)=\operatorname{core}(v) x$ in $\mathbb{M}\left(\Sigma^{ \pm 1}, I\right)$ (it is said that core $(u)$ and core $(v)$ are conjugated in $\mathbb{M}\left(\Sigma^{ \pm 1}, I\right)$ ).

(3) $|\operatorname{core}(u)|_{a}=|\operatorname{core}(v)|_{a}$ for all $a \in \Sigma^{ \pm 1}$ and there exists $k \leq\left|\Sigma^{ \pm 1}\right|$ such that core $(u)$ is a factor of core $(v)^{k}$.

The equivalence of (1) and (2) can be found in [29], the equivalence of (2) and (3) is shown in [16]. We can now infer Theorem 1:

Proof of Theorem 1. Let $\mathbb{A}$ and $\mathbb{B}$ be two given SLPs over $\Sigma^{ \pm 1}$. We want to check, whether $\operatorname{val}(\mathbb{A})$ and $\operatorname{val}(\mathbb{B})$ represent conjugated elements of the graph group $\mathbb{G}(\Sigma, I)$. Using Corollary 23, we can compute in polynomial time SLPs $\mathbb{C}$ and $\mathbb{D}$ with $[\operatorname{val}(\mathbb{C})]_{I}=$ $\operatorname{core}\left([\operatorname{val}(\mathbb{A})]_{I}\right)$ and $[\operatorname{val}(\mathbb{D})]_{I}=\operatorname{core}\left([\operatorname{val}(\mathbb{B})]_{I}\right)$. By Theorem 33, it suffices to check the following two conditions:

- $\left|\operatorname{core}\left([\operatorname{val}(\mathbb{C})]_{I}\right)\right|_{a}=\left|\operatorname{core}\left([\operatorname{val}(\mathbb{D})]_{I}\right)\right|_{a}$ for all $a \in \Sigma^{ \pm 1}$

- There exists $k \leq\left|\Sigma^{ \pm 1}\right|$ such that core $\left([\operatorname{val}(\mathbb{C})]_{I}\right)$ is a factor of core $\left([\operatorname{val}(\mathbb{D})]_{I}\right)^{k}$.

The first condition can be easily checked in polynomial time, since the number of occurrences of a symbol in a compressed strings can be computed in polynomial time. Moreover, the second condition can be checked in polynomial time by Theorem 31, since (by the first condition) we can assume that alph $(\operatorname{val}(\mathbb{C}))=\operatorname{alph}(\operatorname{val}(\mathbb{D}))$. 


\section{Open problems}

Though we have shown that some cases of the simultaneous compressed conjugacy problem for graph groups (see Section 3) can be decided in polynomial time, it remains unclear whether this holds also for the general case. It is also unclear to the authors, whether the general compressed pattern matching problem for traces, where we drop restriction (2), can be decided in polynomial time. Finally, it is not clear, whether Theorem 1-3 also hold if the independence alphabet is part of the input.

\section{References}

1. E. Bach and J. Shallit. Algorithmic Number Theory, volume I: Efficient Algorithms. MIT Press, 1996.

2. R. V. Book and F. Otto. String-Rewriting Systems. Springer, 1993.

3. R. Charney. An introduction to right-angled Artin groups. Geometriae Dedicata, 125:141$158,2007$.

4. R. Charney, J. Crisp, and K. Vogtmann. Automorphisms of 2-dimensional right-angled Artin groups. Geometry \& Topology, 11:2227-2264, 2007.

5. R. Charney and K. Vogtmann. Finiteness properties of automorphism groups of right-angled Artin groups. Bulletin of the London Mathematical Society, 41(1):94-102, 2009.

6. R. Cori, Y. Métivier, and W. Zielonka. Asynchronous mappings and asynchronous cellular automata. Information and Computation, 106(2):159-202, 1993.

7. J. Crisp, E. Godelle, and B. Wiest. The conjugacy problem in right-angled Artin groups and their subgroups. Journal of Topology, 2(3), 2009.

8. M. B. Day. Peak reduction and finite presentations for automorphism groups of right-angled Artin groups. Geometry \& Topology, 13(2):817-855, 2009.

9. V. Diekert. Combinatorics on Traces. Number 454 in Lecture Notes in Computer Science. Springer, 1990.

10. V. Diekert and G. Rozenberg, editors. The Book of Traces. World Scientific, 1995.

11. L. Gasieniec, M. Karpinski, W. Plandowski, and W. Rytter. Efficient algorithms for LempelZiv encoding (extended abstract). In Proceedings of the 5th Scandinavian Workshop on Algorithm Theory (SWAT 1996), number 1097 in Lecture Notes in Computer Science, pages 392-403. Springer, 1996.

12. B. Genest and A. Muscholl. Pattern matching and membership for hierarchical message sequence charts. Theory of Computing Systems, 42(4):536-567, 2008.

13. C. Hagenah. Gleichungen mit regulären Randbedingungen über freien Gruppen. $\mathrm{PhD}$ thesis, University of Stuttgart, Institut für Informatik, 2000.

14. M. R. Laurence. A generating set for the automorphism group of a graph group. Journal of the London Mathematical Society. Second Series, 52(2):318-334, 1995.

15. Y. Lifshits. Processing compressed texts: A tractability border. In Proceedings of the 18th Annual Symposium on Combinatorial Pattern Matching (CPM 2007), number 4580 in Lecture Notes in Computer Science, pages 228-240. Springer, 2007.

16. H.-N. Liu, C. Wrathall, and K. Zeger. Efficient solution to some problems in free partially commutative monoids. Information and Computation, 89(2):180-198, 1990.

17. M. Lohrey. Word problems and membership problems on compressed words. SIAM Journal on Computing, 35(5):1210 - 1240, 2006.

18. M. Lohrey and S. Schleimer. Efficient computation in groups via compression. In Proceedings of Computer Science in Russia (CSR 2007), number 4649 in Lecture Notes in Computer Science, pages 249-258. Springer, 2007. 
19. R. C. Lyndon and P. E. Schupp. Combinatorial Group Theory. Springer, 1977.

20. J. Macdonald. Compressed words and automorphisms in fully residually free groups. International Journal of Algebra and Computation, 2009. to appear.

21. M. Miyazaki, A. Shinohara, and M. Takeda. An improved pattern matching algorithm for strings in terms of straight-line programs. In Proceedings of the 8th Annual Symposium on Combinatorial Pattern Matching (CPM 97), number 1264 in Lecture Notes in Computer Science, pages 1-11. Springer, 1997.

22. A. Myasnikov, V. Shpilrain, and A. Ushakov. Group-based Cryptography. Birkhäuser, 2008.

23. W. Plandowski. Testing equivalence of morphisms on context-free languages. In Second Annual European Symposium on Algorithms (ESA'94), number 855 in Lecture Notes in Computer Science, pages 460-470. Springer, 1994.

24. W. Plandowski and W. Rytter. Application of Lempel-Ziv encodings to the solution of word equations. In Proceedings of the 25th International Colloquium on Automata, Languages and Programming (ICALP 1998), number 1443 in Lecture Notes in Computer Science, pages 731-742. Springer, 1998.

25. W. Plandowski and W. Rytter. Complexity of language recognition problems for compressed words. In J. Karhumäki, H. A. Maurer, G. Paun, and G. Rozenberg, editors, Jewels are Forever, Contributions on Theoretical Computer Science in Honor of Arto Salomaa, pages 262-272. Springer, 1999.

26. S. Schleimer. Polynomial-time word problems. Commentarii Mathematici Helvetici, 83(4):741-765, 2008.

27. H. Servatius. Automorphisms of graph groups. Journal of Algebra, 126(1):34-60, 1989.

28. C. Wrathall. The word problem for free partially commutative groups. Journal of Symbolic Computation, 6(1):99-104, 1988.

29. C. Wrathall. Free partially commutative groups. In Combinatorics, computing and complexity, pages 195-216. Kluwer Academic Press, 1989. 